\title{
Solution of low Mach number aeroacoustic flows using a Variational Multi-Scale finite element formulation of the compressible Navier-Stokes equations written in primitive variables
}

\author{
Camilo Bayona $^{\mathrm{a}, \mathrm{b}}$, Joan Baiges ${ }^{\mathrm{b}, *}$, Ramon Codina ${ }^{\mathrm{a}, \mathrm{b}}$

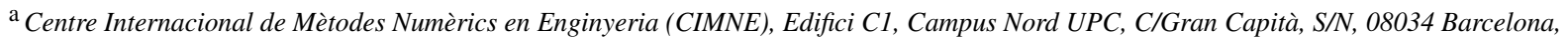 \\ Spain \\ ${ }^{\mathrm{b}}$ Universitat Politècnica de Catalunya, Jordi Girona 1-3, Edifici C1, 08034 Barcelona, Spain
}

Received 28 July 2017; received in revised form 19 January 2018; accepted 22 January 2018

Available online $\mathrm{xxxxx}$

\begin{abstract}
In this work we solve the compressible Navier-Stokes equations written in primitive variables in order to simulate low Mach number aeroacoustic flows. We develop a Variational Multi-Scale formulation to stabilize the finite element discretization by including the orthogonal, dynamic and non-linear subscales, together with an implicit scheme for advancing in time. Three additional features define the proposed numerical scheme: the splitting of the pressure and temperature variables into a relative and a reference part, the definition of the matrix of stabilization parameters in terms of a modified velocity that accounts for the local compressibility, and the approximation of the dynamic stabilization matrix for the time dependent subscales. We also include a weak imposition of implicit non-reflecting boundary conditions in order to overcome the challenges that arise in the aeroacoustic simulations at low compressibility regimes. The order of accuracy of the method is verified for two- and three-dimensional linear and quadratic elements using steady manufactured solutions. Several benchmark flow problems are studied, including transient examples and aeroacoustic applications.
\end{abstract}

(c) 2018 Elsevier B.V. All rights reserved.

Keywords: Compressible flow; Aeroacoustics; Low Mach numbers; Non-reflecting boundary conditions; Variational Multi-Scale (VMS)

\section{Introduction}

The increasing amount of computing resources available in scientific and industrial research has motivated the solution of realistic computational fluid dynamics applications. This is the case of aerodynamics and aeroacoustics; both described by a solution of the compressible Navier-Stokes equations that spans a wide range of scales. An illustrative application of this problem is the simulation of the human voice production inside the mouth, in which the solution of the relevant (small) scales of turbulence and sound waves must be achieved at low compressibility

\footnotetext{
* Corresponding author.

E-mail addresses: cbayona@ cimne.upc.edu (C. Bayona), joan.baiges@upc.edu (J. Baiges), ramon.codina@upc.edu (R. Codina).
} 
conditions. Indeed, compressible turbulent flows and sound waves propagation require an accurate computational method: the small fluctuating scales of sound need to be described, either by higher precision numerical schemes, or by small mesh and time step sizes. In any case, the number of operations required by the computational simulation is very demanding.

Another important requirement for such aeroacoustic problem is that the numerical approximation of the compressible Navier-Stokes equations at low compressibility conditions must be accurate, but many times those methods are not designed to work in these conditions. Most of the compressible flow solvers found in the literature exhibit a degradation in the solution accuracy when the free stream Mach number is progressively reduced. As explained in [1], most of the compressible flow solvers suffer a mismatch between the numerical and the continuous fluxes, and this is principally attributed to the broad difference in length and time scales of the solution. A possible approach for simulating aeroacoustic problems is to solve an aeroacoustic model together with the incompressible Navier-Stokes equations (e.g. reference [2]), but this leads directly to discarding the important physics related to the sound wave production, and consequently, this possibility is discarded in the present article. The type of methods that are intended to be suitable for either compressible or incompressible flows are the so-called unified methods. Among the most remarkable physical models, some modify the incompressible Navier-Stokes equations including extra term (like in the Boussinesq or Low Mach formulations described in [3], and references therein). There are also numerical approximations like [4-8], that aim to be valid at any compressibility regime. These unified approaches lead to more or less accurate descriptions of the fluid flow, but again, they cannot be used in wave propagation problems.

A formulation of the compressible Navier-Stokes equations that can be suitable for the low Mach number limit (or both for compressible and incompressible flows) is important for many reasons. One is that very low Mach numbers can coexist with regions where the flow compressibility becomes considerable. But also, several applications that are traditionally solved with incompressible formulations can be successfully handled by suitable compressible formulations. In this sense, it is important to remark that conservative variables (namely density, momentum, and total energy) are typically used in the compressible formulation, while primitive variables (pressure, velocity, and temperature) are used in the incompressible equations. Including density as a variable in the compressible problem (like in the conservative formulation), results in singularities for the convective terms in the low Mach number limit. Possibilities restrain to primitive and entropic unknowns, which for certain regularity conditions can be shown to satisfy the incompressible equations in the zero Mach number limit. The entropy variable formulation assure a global entropy stability condition, but it is subject to the definition of the entropy function. For more details on entropy variable formulations we refer to $[9,10]$. In the case of the compressible Navier-Stokes equations written in primitive variables, the formulation simplifies to the incompressible equations when the incompressible constraint is included (as explained in [11]), and consequently, it is well defined in the low Mach number limit.

Another particular aspect of the nearly incompressible aeroacoustic flows is that computational boundaries may cause artificial wave reflections related to the ingoing part of the sound waves. Ingoing waves may not only interfere with the acoustic signal relevant to the problem, but they can also produce numerical instabilities if the numerical method is not able to provide enough dissipation [12]. Several approaches to counteract spurious reflections have emerged in the computational aeroacoustics field, among the most popular are: the explicit damping of the compressible equations, the addition of an artificial counter signal, and the solution of non-reflecting boundaries. Damping some terms of the compressible Navier-Stokes equations may be a robust approach that filters the solution in some local regions (called buffer zones), but the computational effort that is required to solve those extra terms is large, and it has led to other approaches, one of which has been to remove the computational domain that is not of prior interest and to solve a non-reflecting boundary condition. The recent literature on non-reflecting boundary conditions is too vast to survey here, but we mention the early work in [13] using asymptotic sets of non-reflecting conditions with pseudo-differential operators, the heuristic radiation arguments in [14], or the asymptotic expansion matching the linearized Euler solution in [15], and refer to the review article [16] for a deeper understanding. Most of non-reflecting boundary methods attempt to damp the incident wave coming from the interior part of the domain.

As commented before, the small fluctuating scales of compressible turbulent flows and sound waves propagation require higher precision numerical schemes in both the temporal and the spatial discretization. This article is focused on the Finite Element Method (FEM) discretization. Among its advantages are the capability of using unstructured meshes to discretize complex geometries, and the possibility of developing efficient high order methods, which are two features highly desired in the Computational Fluid Dynamics (CFD) community. Nonetheless, when the Galerkin method is used to approximate this problem, which possesses non-symmetric operators, an unstable behavior of the 
solution might appear generated, for instance, by unresolved boundary layers. These local instabilities may arise when convection is dominant, and also due to the inf-sup stability condition that restrain the interpolation compatibility of the different variables. Stabilized numerical methods like the Petrov Galerkin Streamline Upwind (SUPG), and the methods that can be framed in the Variational Multi-Scale (VMS) concept, add a stabilization term to the Galerkin formulation, and eliminate both the inf-sup and the convection restrictions. Stabilized finite element methods have been widely applied to both compressible and incompressible formulations, as shown in [17,18], and references therein. See also [19] for a review of VMS methods in CFD. Because only subsonic flows are considered in this study, and more specifically, we restrict the attention to low Mach number flows, the extra control over the gradients of the solution given by discontinuity capturing operators is not required, and consequently, shock capturing techniques are not surveyed here.

In this context, we center the review to the well established VMS stabilization method. This approach splits the unknowns of the problem into a coarse-scale that belongs to the finite element space and a subgrid scale or subscale, which is the remainder. The main idea of the VMS formulation is to include the effect of the unresolved scales in order to stabilize the finite element equations. As a consequence, the correct design of the stabilization operator used to represent the subscales in terms of the resolved scales is crucial for the accuracy and stability of the discretization. Classical stabilized compressible flow formulations, including the compressible Euler equations, give inaccurate results near the low Mach number limit mainly because the standard definitions of the stabilization operator fail to provide adequate numerical diffusion. In order to overcome this difficulty, non diagonal definitions have been proposed (e.g. by [10]). Nonetheless, the diagonal definition can be used with few modifications: this has been the approach taken by [1,20], in which dimensional analysis is used to design a compressible stabilization matrix that is suitable for both compressible and incompressible flows.

In the present work, the primitive variables compressible formulation is used because of its correspondence with the incompressible Navier-Stokes equations in the incompressible limit. Some other improvements are included in the formulation. One important aspect is the use of an implicit time integration scheme in order to avoid the inefficiency that arises from the aeroacoustic propagation in the low Mach number limit. In this sense, and depending on the system of units used for the solution of the problem, the transient problem can include very large quantities in the right-hand-side (RHS) of the continuity and energy equations, which degrade the iterative solution of the discrete linear system. From our perspective, this lack of scaling of the discrete linear system can be healed by splitting the primitive pressure and temperature unknowns into a relative and a reference part. The idea presented here is to change the primitive unknowns to the relative ones, but taking care for including the original unknowns in the non-linear terms of the problem. From the numerical point of view, this transformation not only scales the problem, but also allows to implement iterative linear system solvers in the case of transient problems. We also incorporate some particular features of the VMS framework that we have applied in other flow problems (like in [21,22], for example). Since there are different ways to define the subscales, three different attributes are studied in this work. The first attribute is the definition of the space of the subscales, which can be either the space of finite element residuals, or the space orthogonal to the finite element space [23]. The second attribute is the inclusion of the transient term of the subscale equation [24]. The third attribute is the inclusion of the subscales in all the non-linear terms of the problem [25]. In addition to these definitions, we design the stabilization operator in terms of the local compressibility of the flow, which, in the low Mach number limit, converges to the usual incompressible stabilization operator definition for the continuity and momentum equations. The proposed stabilization operator includes a physical parameter that defines the transition between the compressible and incompressible flow, hence, it combines the features of both stabilized formulations and is capable of describing the range of subsonic Mach numbers.

The focus of the present work is also to investigate the nearly incompressible applications of the compressible flow solver, which involve aeroacoustic applications. For doing so, we implement the characteristic decomposition of waves as a non-reflecting method over the boundaries and prescribe an implicit solution of this method in a weak sense by means of introducing some penalization terms to the compressible formulation. This decomposition of waves, introduced by Thompson in [26], together with the non-reflecting boundary conditions for the NavierStokes equations given by Poinsot and Lele [27], is able to obtain a damped solution at the boundary using a simplification of the conservation equations that are solved inside the computational domain. Instead of solving the compressible Navier-Stokes equation over the boundary, the method annihilates the in-going sound wave by exploiting the characteristics of the Euler equations, where viscous and reaction terms are neglected. Research on annihilating methods for spurious sound wave reflections of the computational boundaries is not the original 
motivation for the present study. More details about the non-reflecting boundary conditions applied to aeroacoustic flow solvers may be found in articles such as [28,29].

The article is organized as follows. We recall the primitive variable formulation of the compressible Navier-Stokes equations in the first part of Section 2. Then we describe the transformation of the primitive variables into a relative and a reference part. Later in that section we present the governing equations for the non-reflecting boundary conditions. In Section 3, we apply the VMS framework in order to discretize in space; we focus our attention to the construction of the stabilization operator in order to properly reach the low Mach number limit. The implicit scheme for advancing in time, and the weak imposition of the non-reflecting conditions are also included in that section. In Section 4, we validate the numerical method for two- and three-dimensional applications by using steady manufactured solutions. We also simulate the differential heated cavity and the flow past a cylinder unsteady problems. Two numerical examples are solved as aeroacoustic applications: the Aeolian tones generated by the flow past a cylinder, and the flow past an open cavity. Finally, we address some conclusions in Section 5.

\section{Governing equations}

In this section we present the compressible Navier-Stokes equations written in primitive variables. For doing this we depart from the conservative formulation, and express the system in terms of the primitive vector of unknowns. The Jacobian and diffusive matrices are therefore written, and the specific formulation for the ideal gas law is included. Then we implement a change of variables, which allows us to scale the discrete linear system. In the last part we describe the governing equations for the non-reflecting boundary conditions.

\subsection{Compressible Navier-Stokes equations written in conservation form}

The problem we consider consists in the compressible Navier-Stokes equations posed in a time interval $\left(0, t_{f}\right)$ and in a domain $\Omega \subset \mathbb{R}^{d}$, being $d$ the number of space dimensions $(d=2$ or 3$)$. Let $t \in\left(0, t_{f}\right)$ be a given time instant in the temporal domain, and $\boldsymbol{x} \in \Omega$ a given point in the spatial domain. Let $\Gamma$ be the boundary of the domain $\Omega$, and $\boldsymbol{n}$ the geometric unit outward normal vector on $\Gamma$. We split $\Gamma$ into two sets: the Dirichlet boundary denoted as $\Gamma_{G}$, and the Neumann boundary denoted as $\Gamma_{N}$. The strong form of the initial and boundary-value problem consists of finding the solution vector $\boldsymbol{U}: \Omega \times\left(0, t_{f}\right) \rightarrow \mathbb{R}^{d+2}$, where $d+2$ is the number of unknowns (the same as equations of the system), such that for the given Dirichlet boundary conditions $\boldsymbol{U}_{G}: \Gamma_{G} \times\left(0, t_{f}\right) \rightarrow \mathbb{R}^{d+2}$ and the Neumann boundary conditions $\boldsymbol{H}: \Gamma_{N} \times\left(0, t_{f}\right) \rightarrow \mathbb{R}^{d+2}$, the following compressible Navier-Stokes equations are satisfied:

$$
\begin{aligned}
\partial_{t} \boldsymbol{U}+\partial_{j} \boldsymbol{E}_{j}(\boldsymbol{U})+\partial_{j} \boldsymbol{G}_{j}(\boldsymbol{U}) & =\boldsymbol{F} & & \text { in } \Omega \subset \mathbb{R}^{d}, \quad t \in\left(0, t_{f}\right), \\
\boldsymbol{D}(\boldsymbol{U}) & =\boldsymbol{D}\left(\boldsymbol{U}_{G}\right) & & \text { on } \Gamma_{G}, \quad t \in\left(0, t_{f}\right), \\
\boldsymbol{B}(\boldsymbol{U}) & =\boldsymbol{H} & & \text { on } \Gamma_{N}, \quad t \in\left(0, t_{f}\right), \\
\boldsymbol{U} & =\boldsymbol{U}^{0}(\boldsymbol{x}) & & \text { in } \Omega \subset \mathbb{R}^{d}, \quad t=0 .
\end{aligned}
$$

Here $\partial_{t}$ and $\partial_{j}$ are short notations that indicate the Eulerian time derivative and $\partial / \partial x_{j}$, respectively. The usual summation convention is implied in the equation presented before, with indices running from 1 to $d$. The Dirichlet boundary operator $\boldsymbol{D}(\cdot)$ is used to impose the components of $\boldsymbol{U}$ on different parts of $\Gamma$. The Neumann boundary conditions are given by the operator $\boldsymbol{B}(\cdot)$. Note that with our notation $\Gamma_{G}$ and $\Gamma_{N}$ may overlap.

The vector of conservative variables $\boldsymbol{U}$, the convective flux in the $j$ th-direction $\boldsymbol{E}_{j}$, the diffusive flux in the $j$ thdirection $\boldsymbol{G}_{j}$, and the vector of forces $\boldsymbol{F}$ are defined as:

$$
\begin{array}{cc}
\boldsymbol{U}=\left[\begin{array}{c}
\rho \\
\rho u_{1} \\
\rho u_{2} \\
\rho u_{3} \\
e_{\mathrm{tot}}
\end{array}\right], & \boldsymbol{E}_{j}(\boldsymbol{U})=\left[\begin{array}{c}
\rho u_{j} \\
\rho u_{j} u_{1}+p \delta_{1 j} \\
\rho u_{j} u_{2}+p \delta_{2 j} \\
\rho u_{j} u_{3}+p \delta_{3 j} \\
u_{j}\left(e_{\mathrm{tot}}+p\right)
\end{array}\right], \\
\boldsymbol{G}_{j}(\boldsymbol{U})=\left[\begin{array}{c}
0 \\
-\tau_{j 1} \\
-\tau_{j 2} \\
-\tau_{j 3} \\
-u_{i} \tau_{i j}+q_{j}
\end{array}\right], & \boldsymbol{F}=\left[\begin{array}{c}
0 \\
\rho \boldsymbol{f} \\
\rho \boldsymbol{f} \cdot \boldsymbol{u}+\rho r
\end{array}\right] .
\end{array}
$$


In these relations $\rho$ is the density, $p$ is the pressure, $\boldsymbol{u}$ is the velocity, $e_{\text {tot }}$ is the total energy, defined as $e_{\text {tot }}=$ $\rho(e+\boldsymbol{u} \cdot \boldsymbol{u} / 2), e$ being the internal energy, $\boldsymbol{f}$ is a body force vector, $r$ is a heat source/sink term, $\boldsymbol{I}=\left[\delta_{i j}\right]$ is the identity or Kronecker tensor, $\boldsymbol{\tau}$ is the viscous stress tensor, which for a Newtonian fluid is defined as

$$
\tau_{i j}(\boldsymbol{u})=\mu\left(\partial_{j} u_{i}+\partial_{i} u_{j}\right)-\frac{2 \mu}{3}\left(\partial_{l} u_{l}\right) \delta_{i j}
$$

and $\boldsymbol{q}$ is the heat flux vector, that we calculate using Fourier's law as

$$
q_{i}(T)=-\lambda \partial_{i} T,
$$

where $\mu$ is the viscosity, $\lambda$ is the thermal conductivity, and $T$ is the temperature of the fluid. The caloric equation $e=c_{v}(T) T$ and the ideal law for gases $p=\rho R T$ are used to calculate the pressure and the acoustic speed $c$. In these relations the specific heat at constant volume $c_{v}(T)$ and the specific heat at constant pressure $c_{p}(T)$ are thermodynamic properties of the fluid. We also define $\gamma=c_{p} / c_{v}$ for the ratio between the specific heats, and $R=c_{p}-c_{v}$ for the specific gas constant. The non-dimensional Mach number $\mathrm{M}=|\boldsymbol{u}| / c$ is used to calculate the compressibility regime.

\subsection{Compressible Navier-Stokes equations written in primitive variables}

In order to formulate the equations using primitive variables, we express the conservative unknowns $\boldsymbol{U}$ as a function of the primitive variables $\boldsymbol{Y}=(p, \boldsymbol{u}, T)^{\top}$. Hereafter, let us denote the transpose operation by the superscript $\top$. Hence, Eq. (1) can be written as

$$
\partial_{t} \boldsymbol{U}(\boldsymbol{Y})+\partial_{j} \boldsymbol{E}_{j}(\boldsymbol{U}(\boldsymbol{Y}))+\partial_{j} \boldsymbol{G}_{j}(\boldsymbol{U}(\boldsymbol{Y}))=\boldsymbol{F} .
$$

If $\boldsymbol{Y}$ is sufficiently smooth, it also satisfies the quasi-linear form

$$
\boldsymbol{A}_{0}(\boldsymbol{Y}) \partial_{t} \boldsymbol{Y}+\boldsymbol{A}_{j}(\boldsymbol{Y}) \partial_{j} \boldsymbol{Y}-\partial_{k}\left(\boldsymbol{K}_{k j}(\boldsymbol{Y}) \partial_{j} \boldsymbol{Y}\right)=\boldsymbol{F} .
$$

Here we have written the divergence of the fluxes in Eq. (8) in a more convenient manner by defining the Jacobian matrices $\boldsymbol{A}_{0}(\boldsymbol{Y})=\partial \boldsymbol{U} / \partial \boldsymbol{Y}, \boldsymbol{A}_{j}(\boldsymbol{Y})=\partial \boldsymbol{E}_{j}(\boldsymbol{U}(\boldsymbol{Y})) / \partial \boldsymbol{Y}$, and the diffusivity matrix $\boldsymbol{K}(\boldsymbol{Y})=\left[\boldsymbol{K}_{k j}(\boldsymbol{Y})\right]$, such that $\partial_{j} \boldsymbol{G}_{j}(\boldsymbol{U}(\boldsymbol{Y}))=-\partial_{k}\left(\boldsymbol{K}_{k j}(\boldsymbol{Y}) \partial_{j} \boldsymbol{Y}\right)$.

In order to express all the previous relations in terms of the primitive variables alone, we make the supposition that the fluid is divariant and account for the ideal gas law. This makes possible to formulate density as a function of the pressure and the temperature, and to rearrange the Navier-Stokes equations (see [11,30]). Hence, the Jacobian matrices $\boldsymbol{A}_{j}(\boldsymbol{Y}), j=0, \ldots, d$, can be formulated as functions of primitive variables and thermodynamic coefficients:

$$
\begin{aligned}
\boldsymbol{A}_{0}(\boldsymbol{Y})= & {\left[\begin{array}{ccc}
\rho \beta_{t} & \mathbf{0}^{\top} & -\rho \alpha_{p} \\
\rho \beta_{t} \boldsymbol{u} & \rho \boldsymbol{I} & -\rho \alpha_{p} \boldsymbol{u} \\
\rho \beta_{t} a_{1}-\alpha_{p} T & \rho \boldsymbol{u}^{\top} & -\rho \alpha_{p} a_{1}+\rho c_{p}
\end{array}\right], } \\
\boldsymbol{A}_{j}(\boldsymbol{Y})= & {\left[\begin{array}{ccc}
\rho \beta_{t} u_{j} & \rho \boldsymbol{e}_{j}^{\top} & -\rho \alpha_{p} u_{j} \\
\rho \beta_{t} \boldsymbol{u} u_{j}+\boldsymbol{e}_{j} & \rho \boldsymbol{I} u_{j}+\rho \boldsymbol{u} \otimes \boldsymbol{e}_{j} & -\rho \alpha_{p} \boldsymbol{u} u_{j} \\
\left(\rho \beta_{t} a_{1}-\alpha_{p} T+1\right) u_{j} & \rho \boldsymbol{u}^{\top} u_{j}+\rho a_{1} \boldsymbol{e}_{j}^{\top} & \left(-\rho \alpha_{p} a_{1}+\rho c_{p}\right) u_{j}
\end{array}\right], }
\end{aligned}
$$

for $j=1, \ldots, d$, . Hereafter $\mathbf{0}$ is the vector of $\mathbb{R}^{d}$ with zero in all its components. The thermodynamic relation $a_{1}$ stands for $a_{1}=c_{v} T+p / \rho+|\boldsymbol{u}|^{2} / 2, \alpha_{p}$ is the volume expansivity, and $\beta_{t}$ is the isothermal compressibility, given by

$$
\alpha_{p}=-\frac{1}{\rho}\left(\frac{\partial \rho}{\partial T}\right)_{p} \quad \text { and } \quad \beta_{t}=\frac{1}{\rho}\left(\frac{\partial \rho}{\partial p}\right)_{T}
$$

with $(\cdot)_{p}$ and $(\cdot)_{T}$ defining a constant pressure and temperature constrain, respectively. Additionally, the diffusive matrix $\boldsymbol{K}_{k j}(\boldsymbol{Y})$ is constructed as

$$
\boldsymbol{K}_{j j}(\boldsymbol{Y})=\left[\begin{array}{ccc}
0 & \mathbf{0}^{\top} & 0 \\
\mathbf{0} & \mu \boldsymbol{I}+\frac{1}{3} \mu \boldsymbol{e}_{j} \otimes \boldsymbol{e}_{j} & \mathbf{0} \\
0 & \mu \boldsymbol{u}^{\top}+\frac{1}{3} \mu u_{j} \boldsymbol{e}_{j}^{\top} & \lambda
\end{array}\right] \quad \text { for } j=1, \ldots, d, \text { and }
$$




$$
\boldsymbol{K}_{k j}(\boldsymbol{Y})=\left[\begin{array}{ccc}
0 & \mathbf{0}^{\top} & 0 \\
\mathbf{0} & \mu \boldsymbol{e}_{j} \otimes \boldsymbol{e}_{k}-\frac{2}{3} \mu \boldsymbol{e}_{k} \otimes \boldsymbol{e}_{j} & \mathbf{0} \\
0 & \mu u_{j} \boldsymbol{e}_{k}^{\top}-\frac{2}{3} \mu u_{k} \boldsymbol{e}_{j}^{\top} & 0
\end{array}\right] \quad \text { for } k, j=1, \ldots, d, \text { with } k \neq j
$$

In all these expressions, we have denoted by $\boldsymbol{e}_{i}$ the unit vector in the $i$ th direction.

In this work the ideal law for gases is used, so that expressions (10) result in $\alpha_{p}=1 / T$, and $\beta_{t}=1 / p$. For ideal gases at the low Mach number limit, the fluid exhibits very large values of pressure and temperature. As a consequence, the volume expansivity and the isothermal compressibility tend to zero, and the Jacobian matrices recover the usual incompressible form. The divergence free terms present in both the convective and diffusive matrices constrain the incompressibility of the fluid in this regime. All the matrices stay bounded when the Mach number tends to zero because the density remains almost constant.

\subsubsection{Relative variable formulation}

As stated before, using the primitive formulation for ideal gases in the low Mach number limit results in having to operate with very large quantities in the continuity and energy equations (e.g. if the international system of units is used for typical gases). When an implicit numerical scheme is used, the linear system that needs to be solved at each time step contains very large values for these two equations, making it very inefficient to solve the discrete linear system by using iterative methods. In order to overcome this difficulty, we perform a decomposition of the primitive (absolute) variables $\boldsymbol{Y}$, into a relative (or gauge) part $\boldsymbol{Y}^{*}$, and a reference (or atmospheric) part $\boldsymbol{Y}_{\mathrm{atm}}$ :

$$
\underbrace{\left[\begin{array}{l}
p \\
\boldsymbol{u} \\
T
\end{array}\right]}_{\boldsymbol{Y}}=\underbrace{\left[\begin{array}{c}
p^{*} \\
\boldsymbol{u}^{*} \\
T^{*}
\end{array}\right]}_{\boldsymbol{Y}^{*}}+\underbrace{\left[\begin{array}{c}
p_{\mathrm{atm}} \\
\mathbf{0} \\
T_{\mathrm{atm}}
\end{array}\right]}_{\boldsymbol{Y} \mathrm{atm}},
$$

supposing that the reference part is constant. Then, we write Eq. (9) by using the relative variables as the unknowns, but caring to take into account the complete contribution for calculating non-linear terms; we have

$$
\boldsymbol{A}_{0}(\boldsymbol{Y}) \partial_{t} \boldsymbol{Y}^{*}+\boldsymbol{A}_{j}(\boldsymbol{Y}) \partial_{j} \boldsymbol{Y}^{*}-\partial_{k}\left(\boldsymbol{K}_{k j}(\boldsymbol{Y}) \partial_{j} \boldsymbol{Y}^{*}\right)=\boldsymbol{F}
$$

Moreover, the previous equation can be written for convenience as

$$
\boldsymbol{A}_{0}(\boldsymbol{Y}) \partial_{t} \boldsymbol{Y}^{*}+\mathcal{L}\left(\boldsymbol{Y} ; \boldsymbol{Y}^{*}\right)=\boldsymbol{F},
$$

by introducing the nonlinear operator

$$
\mathcal{L}\left(\boldsymbol{Y} ; \boldsymbol{Y}^{*}\right)=\boldsymbol{A}_{j}(\boldsymbol{Y}) \partial_{j} \boldsymbol{Y}^{*}-\partial_{k}\left(\boldsymbol{K}_{k j}(\boldsymbol{Y}) \partial_{j} \boldsymbol{Y}^{*}\right)
$$

which includes the definitions for the convective and diffusive terms. Initial and boundary conditions must be set for the primitive problem written using relative unknowns. We explicitly indicate in our examples how these are prescribed.

\subsection{Weak form of the problem}

Let $\mathcal{W}$ be the space of functions where, for each $t \in\left(0, t_{f}\right)$, the unknowns are well defined, with appropriate regularity that we will not analyze here. Let us also denote by $(\cdot, \cdot)_{\omega}$ the integral of the product of two functions (scalar or vector valued) in a domain $\omega$, omitting the subscript when $\omega=\Omega$. Introducing the form

$$
A(\boldsymbol{U} ; \boldsymbol{V}, \boldsymbol{W}):=\left(\boldsymbol{V}, \boldsymbol{A}_{j}(\boldsymbol{U}) \partial_{j} \boldsymbol{W}\right)+\left(\partial_{k} \boldsymbol{V}, \boldsymbol{K}_{k j}(\boldsymbol{U}) \partial_{j} \boldsymbol{W}\right),
$$

the variational form of the problem can be written as: find $\boldsymbol{Y}^{*}:\left(0, t_{f}\right) \rightarrow \mathcal{W}$ such that

$$
\begin{aligned}
\left(\boldsymbol{V}, \boldsymbol{A}_{0}(\boldsymbol{Y}) \partial_{t} \boldsymbol{Y}^{*}\right)+A\left(\boldsymbol{Y} ; \boldsymbol{V}, \boldsymbol{Y}^{*}\right) & =(\boldsymbol{V}, \boldsymbol{F})+(\boldsymbol{V}, \boldsymbol{H})_{\Gamma_{N}}, & & t \in\left(0, t_{f}\right), \\
(\boldsymbol{V}, \boldsymbol{Y}) & =\left(\boldsymbol{V}, \boldsymbol{Y}^{0}\right), & & t=0,
\end{aligned}
$$

for all $\boldsymbol{V}$ in the adequate test functions space. In this case, the Neumann boundary operator is given by the diffusive fluxes

$$
\boldsymbol{B}(\boldsymbol{Y})=-n_{j} \boldsymbol{K}_{k j}(\boldsymbol{Y}) \partial_{j} \boldsymbol{Y}^{*}
$$


although part of the convective term could also be integrated by parts and contribute to the Neumann boundary conditions, in particular, the pressure term.

\subsection{Non-reflecting boundary conditions}

Another particular aspect of the nearly incompressible limit is that the computational boundaries, essentially the subsonic outlets, may cause artificial wave reflections related to the ingoing part of the propagated sound wave. In order to overcome this difficulty we incorporate non-reflecting boundary conditions to the compressible formulation.

\subsubsection{Local one-dimensional characteristic wave equation}

Specifically, the characteristic boundary conditions for the Navier-Stokes equations by Poinsot and Lele [27] are implemented to counteract spurious reflections. The characteristic boundary conditions aim to compute the flow at the boundary using an approximation of the governing equations that are solved inside the computational domain. This method employs the hypothesis that, as waves are associated with the hyperbolic part of the Navier-Stokes equations, not with the diffusive part, the flow at the boundary can be approximated as inviscid and one-dimensional. Hence, the Euler equations can be represented as a local one-dimensional characteristic wave equation.

The characteristics analysis is related to the flow description at the boundary in local coordinates instead of using the interior domain coordinates. Considering $d=3$, a transformation from the computational domain with Cartesian coordinates $\left(x_{1}, x_{2}, x_{3}\right)$, to a local reference frame with coordinates $(\xi, \eta, \zeta)$, is performed to determine the physical waves and their orientation at the boundary. In the new local reference system of coordinates, $\xi$ defines the normal direction to the boundary $\Gamma$, and $\eta$ and $\zeta$ define tangential directions.

According to the characteristic analysis performed by Thompson [26] over the Euler equations, these can be decomposed using the primitive variables (including the definition of the $u_{i}$ flow velocity in the local reference frame $\left.\boldsymbol{u}=\left(u_{\xi}, u_{\eta}, u_{\zeta}\right)^{\top}\right)$, with the axial derivatives grouped into a constant source term, so that, the convective term can be diagonalized using a similarity transformation. The resulting nonlinear convective problem is then written as follows:

$$
\begin{aligned}
\frac{\partial p^{*}}{\partial t}+\frac{1}{2}\left(\chi_{5}+\chi_{1}\right) & =0, \\
\frac{\partial u_{\xi}}{\partial t}+\frac{1}{2 \rho c}\left(\chi_{5}-\chi_{1}\right) & =0, \\
\frac{\partial u_{\eta}}{\partial t}+\chi_{3} & =0, \\
\frac{\partial u_{\zeta}}{\partial t}+\chi_{4} & =0, \\
\frac{\partial T^{*}}{\partial t}+\frac{T}{\rho c^{2}}\left[-\chi_{2}+\frac{(\gamma-1)}{2}\left(\chi_{5}+\chi_{1}\right)\right] & =0 .
\end{aligned}
$$

Here $\chi_{i}$, stands for the amplitude variations of the characteristic $i$ th wave, $i=1,2,3,4,5$. Those variations are calculated in terms of normal derivatives to the boundary as follows:

$$
\chi=\left(\begin{array}{c}
\lambda_{1}\left(\frac{\partial p^{*}}{\partial \xi}-\rho c \frac{\partial u_{\xi}}{\partial \xi}\right) \\
\lambda_{2}\left(c^{2} \frac{\partial \rho}{\partial \xi}-\frac{\partial p^{*}}{\partial \xi}\right) \\
\lambda_{3} \frac{\partial u_{\eta}}{\partial \xi} \\
\lambda_{4} \frac{\partial u_{\zeta}}{\partial \xi} \\
\lambda_{5}\left(\frac{\partial p^{*}}{\partial \xi}+\rho c \frac{\partial u_{\xi}}{\partial \xi}\right)
\end{array}\right),
$$

where $\lambda_{i}$ are the characteristic velocities:

$$
\begin{aligned}
& \lambda_{1}=u_{\xi}-c, \\
& \lambda_{2}=\lambda_{3}=\lambda_{4}=u_{\xi}, \\
& \lambda_{5}=u_{\xi}+c .
\end{aligned}
$$


Therefore, the characteristics determine at each boundary which waves associated to those values are incoming or outgoing. The validity of this method, which has been pointed out in [31], is subject to the consideration that the characteristic waves represent a one-dimensional propagation in the stream-wise direction. As no genuine characteristics exist for multi-dimensional problems, these equations make a raw approximation of the wave propagation phenomena at the boundary. Because obliquely incident waves are neglected, the accuracy of the method must be kept with the appropriate design of the boundary (as done in the numerical examples to be presented next in the article), so that perpendicular waves impinging the non-reflecting boundaries are privileged.

Again, we propose to use a vector of primitive variables at the boundary $\boldsymbol{J}=\left(p, u_{\xi}, u_{\eta}, u_{\zeta}, T\right)^{\top}$, which is written conveniently by using the local reference system, but also including the decomposition of the unknowns into a relative and an absolute part. For convenience we introduce a general form, in which Eqs. (17)-(21) can be written, that contains additional terms as explained next. We write the boundary problem in vector form as follows:

$$
\partial_{t} \boldsymbol{J}^{*}+\boldsymbol{A}_{B}(\boldsymbol{J}) \frac{\partial \boldsymbol{J}^{*}}{\partial \xi}+\boldsymbol{S}_{B}(\boldsymbol{J})\left(\boldsymbol{J}^{*}-\widehat{\boldsymbol{J}}^{*}\right)=\boldsymbol{F}_{B} \quad \text { in } \quad \Gamma \subset \mathbb{R}^{d}, \quad t \in\left(0, t_{f}\right) .
$$

This arrangement makes possible to deal with the non-linearities of the problem, and to separate the equation to be solved into a transient, a convective-like, a reactive, and a forcing term. The convective term is written as a non-linear $(d+2) \times(d+2)$ convective matrix $\boldsymbol{A}_{B}(\boldsymbol{J})$ multiplied by the normal derivative of the unknowns at the boundary. The introduction of the reactive and forcing terms allows to deal with the non-reflecting outflow condition (that is explained in detail in the next part). In this sense, we construct the reactive term as a non-linear reactive matrix $\boldsymbol{S}_{B}(\boldsymbol{J})$ that multiplies the perturbed solution at the boundary $\left(\boldsymbol{J}^{*}-\widehat{\boldsymbol{J}}^{*}\right)$, being $\widehat{\boldsymbol{J}}^{*}=\left(p_{\infty}^{*}, u_{\xi \infty}, u_{\eta \infty}, u_{\zeta \infty}, T_{\infty}^{*}\right)^{\top}$ the unperturbed solution at the far-field. Moreover, the convective, and reactive terms are also grouped into the non-linear boundary operator

$$
\mathcal{L}_{B}\left(\boldsymbol{J} ; \boldsymbol{J}^{*}\right)=\boldsymbol{A}_{B}(\boldsymbol{J}) \frac{\partial \boldsymbol{J}^{*}}{\partial \xi}+\boldsymbol{S}_{B}(\boldsymbol{J}) \boldsymbol{J}^{*} .
$$

The boundary problem can be solved without difficulty by transforming back (26) into a problem which possesses $\boldsymbol{Y}$ as unknowns. This is achieved using the transformation matrix $\boldsymbol{T}$ written in system form between the original reference system and the local boundary system:

$$
\boldsymbol{T}=\left[\begin{array}{ccccc}
1 & 0 & 0 & 0 & 0 \\
0 & T_{\xi x_{1}} & T_{\xi x_{2}} & T_{\xi x_{3}} & 0 \\
0 & T_{\eta x_{1}} & T_{\eta x_{2}} & T_{\eta x_{3}} & 0 \\
0 & T_{\zeta x_{1}} & T_{\zeta x_{2}} & T_{\zeta x_{3}} & 0 \\
0 & 0 & 0 & 0 & 1
\end{array}\right] .
$$

At this point, let us note that, if $\boldsymbol{Y}^{*}$ is an element in the domain of $\mathcal{L}\left(\boldsymbol{Y} ; \boldsymbol{Y}^{*}\right)$ and $\boldsymbol{H}$ is an element in its range, and, if $\boldsymbol{J}^{*}$ is an element in the domain of $\mathcal{L}_{B}\left(\boldsymbol{J}, \boldsymbol{J}^{*}\right)$ and $\boldsymbol{F}_{B}$ an element in its range, the unknowns and force vectors can be transformed respectively as

$$
\boldsymbol{Y}^{*}=\boldsymbol{T}^{-1} \boldsymbol{J}^{*} \quad \text { and } \quad \boldsymbol{H}=\boldsymbol{T}^{-1} \boldsymbol{F}_{B} .
$$

Here we employ the fact that the derivatives of $\boldsymbol{T}$ and $\boldsymbol{T}^{-1}$ are null, and that the solution is sufficiently smooth. Consequently, the local boundary problem can be transformed onto the original reference system. For convenience we multiply both sides of the transformed boundary problem with the transient Jacobian matrix $\boldsymbol{A}_{0}(\boldsymbol{Y})$, and thus the problem is now written as

$$
\begin{aligned}
\boldsymbol{A}_{0}(\boldsymbol{Y}) \partial_{t} \boldsymbol{Y}^{*}+\boldsymbol{A}_{0}(\boldsymbol{Y}) \boldsymbol{T}^{-1} \mathcal{L}_{B}\left(\boldsymbol{J}, \boldsymbol{T} \boldsymbol{Y}^{*}\right)= \\
\boldsymbol{A}_{0}(\boldsymbol{Y}) \boldsymbol{T}^{-1} \boldsymbol{F}_{B}+\boldsymbol{A}_{0}(\boldsymbol{Y}) \boldsymbol{T}^{-1} \boldsymbol{S}_{B}(\boldsymbol{J}) \widehat{\boldsymbol{J}}^{*} \quad \text { in } \quad \Gamma \subset \mathbb{R}^{d} .
\end{aligned}
$$

\subsubsection{Non-reflecting subsonic outflow}

Non-reflecting boundaries are constructed by taking control over the amplitude variations of the characteristic waves. In particular, for subsonic outlets, the velocity of sound propagation waves along the negative and positive stream-wise directions $\lambda_{1}$ and $\lambda_{5}$ are crucial. An appropriate non-reflecting boundary condition for this case must set 
the value of the wave amplitude variation $\chi_{1}$ associated to the characteristic velocity $\lambda_{1}=u_{\xi}-c$, which is the ingoing part of the wave. In this work we implement the Rudy and Strikwerda relation [14]:

$$
\chi_{1}=\sigma c \frac{\left(1-\mathrm{M}^{2}\right)}{l}\left(p^{*}-p_{\infty}^{*}\right),
$$

in order to calculate the incoming wave amplitude variation at the subsonic outflow. In the previous relation $\sigma$ stands for a pressure relaxation parameter, $l$ is the characteristic length-scale of the propagation domain, and $p_{\infty}^{*}$ is the unperturbed value for pressure. At low Mach numbers, the previous relation simplifies to a scaled pressure relaxation parameter multiplying the pressure perturbation.

For the three-dimensional non-reflecting subsonic outflow, matrices $\boldsymbol{A}_{B}(\boldsymbol{J})$, and $\boldsymbol{S}_{B}(\boldsymbol{J})$ are defined respectively as

$$
\boldsymbol{A}_{B}(\boldsymbol{J})=\left[\begin{array}{ccccc}
\frac{1}{2}\left(u_{\xi}+c\right) & \frac{\rho c}{2}\left(u_{\xi}+c\right) & 0 & 0 & 0 \\
\frac{1}{2 \rho c}\left(u_{\xi}+c\right) & \frac{1}{2}\left(u_{\xi}+c\right) & 0 & 0 & 0 \\
0 & 0 & u_{\xi} & 0 & 0 \\
0 & 0 & 0 & u_{\xi} & 0 \\
\frac{T}{\rho c^{2}}\left[\frac{1}{2}(\gamma-1)\left(u_{\xi}+c\right)+u_{\xi}\right]-\frac{u_{\xi}}{\rho R} & \frac{T}{2 c}(\gamma-1)\left(u_{\xi}+c\right) & 0 & 0 & u_{\xi}
\end{array}\right],
$$

and

$$
\boldsymbol{S}_{B}(\boldsymbol{J})=\left[\begin{array}{ccccc}
\sigma c \frac{\left(1-M^{2}\right)}{2 l} & 0 & 0 & 0 & 0 \\
-\sigma \frac{\left(1-M^{2}\right)}{2 \rho l} & 0 & 0 & 0 & 0 \\
0 & 0 & 0 & 0 & 0 \\
0 & 0 & 0 & 0 & 0 \\
\frac{T \sigma(\gamma-1)\left(1-M^{2}\right)}{2 \rho c l} & 0 & 0 & 0 & 0
\end{array}\right] .
$$

The forcing term contains zero in all its $(d+2)$ components.

Note that for this non-reflecting condition, $\chi_{1}$ is the only amplitude variation that is modeled (using (29) instead of calculating it with (22)). The remaining components of $\chi$ are calculated from relation (22).

\subsubsection{Non-reflecting subsonic inflow}

In the case of a non-reflecting subsonic inflow, four characteristic waves are entering the domain $\left(\chi_{1}, \chi_{2}, \chi_{3}\right.$, and $\chi_{4}$ ), and only one $\left(\chi_{5}\right)$ is leaving. This type of boundary condition relies on the imposition of (the given) Dirichlet conditions for velocity and temperature, but calculates the pressure depending on the outgoing wave crossing the boundary. Since $\chi_{1}$ and $\chi_{5}$ are the characteristic waves involved in the pressure equation, the method calculates the outgoing amplitude $\chi_{5}$ from the interior domain, and controls $\chi_{1}$ (related to the reflection) by using the following equation obtained from (18):

$$
\chi_{1}=\chi_{5}+2 \rho c \frac{\partial u_{\xi}}{\partial t} .
$$

Hence, using Eq. (17) the resulting equation for pressure is such that

$$
\frac{\partial p^{*}}{\partial t}+\left(u_{\xi}+c\right)\left(\frac{\partial p^{*}}{\partial \xi}+\rho c \frac{\partial u_{\xi}}{\partial \xi}\right)=-\rho c \frac{\partial u_{\xi}}{\partial t} .
$$

In this work we only consider constant and homogeneous inlet velocities. Therefore, the RHS of the previous equation vanishes, and the components of $\boldsymbol{F}_{B}$ are identically zero. In the case of the $\boldsymbol{A}_{B}(\boldsymbol{J})$ matrix, it is composed by $\left(u_{\xi}+c\right)$ in the first position of the first row, and by $\rho c\left(u_{\xi}+c\right)$ in the second position of the first row. The rest of terms are zero. Finally, the $S_{B}(J)$ matrix contains zero in all its components.

\section{Numerical methods}

In the previous section we have developed a compressible Navier-Stokes formulation conceived for ideal gases, which cares for splitting the primitive unknowns into a relative and an absolute part. The resulting model is designed to be suitable at the low Mach number limit. Non-reflecting boundary conditions have been also described for solving aeroacoustic applications. As explained before, we discretize Eqs. (12) and (28) in space using the finite element 
method. However, when the Galerkin method is used to approximate the Navier-Stokes equations (which possess non-symmetric operators), an unstable behavior of the solution might appear when convection is dominant, and due to the incompatibility between the interpolation spaces of the different variables.

In this section we recall the VMS framework, and apply it to the compressible flow equations in order to stabilize the finite element formulation. We then explain the particular features of the VMS method that we include in the numerical formulation. Later, we construct the approximation of the stabilization matrix that is suitable for the low Mach number limit. In the present article, the extra diffusion that is given by shock capturing methods (see [18]) is not needed because our interest is restricted to low Mach regimes. At the end of this section, we present the implicit scheme for advancing in time the compressible equations, the approximation for the time tracking of the subscales, and the numerical scheme that we apply to obtain the solution at the non-reflecting boundaries.

\subsection{The space discrete variational multi-scale stabilized finite element formulation}

Let us first consider a finite-element partition $\mathcal{T}_{h}=\{K\}$ of the domain $\Omega$. The diameter of the element partition is denoted by $h$. We define the finite element space $\mathcal{W}_{h} \subset \mathcal{W}$ as made of continuous piecewise polynomial functions in space. The Galerkin approximation to problem (15)-(16) can be stated as follows: find $\boldsymbol{Y}_{h}^{*}:\left(0, t_{f}\right) \rightarrow \mathcal{W}_{h}$ such that

$$
\begin{aligned}
\left(\boldsymbol{V}_{h}, \boldsymbol{A}_{0}\left(\boldsymbol{Y}_{h}\right) \partial_{t} \boldsymbol{Y}_{h}^{*}\right)+A\left(\boldsymbol{Y}_{h} ; \boldsymbol{V}_{h}, \boldsymbol{Y}_{h}\right) & =\left(\boldsymbol{V}_{h}, \boldsymbol{F}\right)+\left(\boldsymbol{V}_{h}, \boldsymbol{H}\right)_{\Gamma_{N}}, & & t \in\left(0, t_{f}\right), \\
\left(\boldsymbol{V}_{h}, \boldsymbol{Y}_{h}\right) & =\left(\boldsymbol{V}_{h}, \boldsymbol{Y}^{0}\right), & & t=0,
\end{aligned}
$$

for all $\boldsymbol{V}_{h} \in \mathcal{W}_{h}^{0}$, the discrete space of test functions (i.e., with components vanishing where Dirichlet conditions are prescribed on the boundary).

This approximation suffers from instability problems, which vary according to the way to construct $\mathcal{W}_{h}$ (e.g. in the case of equally interpolating spaces), and to the weight of the non-linear convective term.

We stabilize the compressible Navier-Stokes equations based on the VMS approach introduced in [17]. The basic idea is to approximate the effect of the components of the solution of the continuous problem that cannot be solved by the finite element mesh. The method decomposes the space of the unknowns into a finite-dimensional space $\mathcal{W}_{h}$, and an infinite-dimensional one, $\widetilde{\mathcal{W}}$, so that $\mathcal{W}=\mathcal{W}_{h} \oplus \widetilde{\mathcal{W}}$. The unknown and the test functions are accordingly split as $\boldsymbol{Y}^{*}=\boldsymbol{Y}_{h}^{*}+\widetilde{\boldsymbol{Y}}^{*}$ and $\boldsymbol{V}=\boldsymbol{V}_{h}+\widetilde{\boldsymbol{V}}$, respectively. Eq. (15) can be equivalently written as the system of equations

$$
\left(\boldsymbol{V}_{h}, \boldsymbol{A}_{0}(\boldsymbol{Y}) \partial_{t} \boldsymbol{Y}^{*}\right)+A\left(\boldsymbol{Y} ; \boldsymbol{V}_{h}, \boldsymbol{Y}^{*}\right)=\left(\boldsymbol{V}_{h}, \boldsymbol{F}\right)+\left(\boldsymbol{V}_{h}, \boldsymbol{H}\right)_{\Gamma_{N}},
$$

for all $\boldsymbol{V}_{h} \in \mathcal{W}_{h}^{0}, t \in\left(0, t_{f}\right)$, and

$$
\left(\widetilde{\boldsymbol{V}}, \boldsymbol{A}_{0}(\boldsymbol{Y}) \partial_{t} \boldsymbol{Y}^{*}\right)+A(\boldsymbol{U} ; \tilde{\boldsymbol{V}}, \boldsymbol{U})=(\tilde{\boldsymbol{V}}, \boldsymbol{F})+(\widetilde{\boldsymbol{V}}, \boldsymbol{H})_{\Gamma_{N}},
$$

for all $\widetilde{\boldsymbol{V}} \in \widetilde{\mathcal{W}}^{0}, t \in\left(0, t_{f}\right)$, and likewise for the initial condition, Eq. (16). In Eq. (35), $\widetilde{\mathcal{W}}^{0}$ is the space of subscale test functions.

\subsubsection{Finite element equation}

We first analyze the equation for the finite element scale. The second term in the left-hand-side (LHS) of Eq. (34) can be split as

$$
A\left(\boldsymbol{Y} ; \boldsymbol{V}_{h}, \boldsymbol{Y}^{*}\right)=A_{G}\left(\boldsymbol{Y} ; \boldsymbol{V}_{h}, \boldsymbol{Y}_{h}^{*}\right)+A_{S}\left(\boldsymbol{Y} ; \boldsymbol{V}_{h}, \widetilde{\boldsymbol{Y}}^{*}\right)
$$

defining the Galerkin terms related to the application of the non-linear operator to the finite element unknown as

$$
A_{G}\left(\boldsymbol{Y} ; \boldsymbol{V}_{h}, \boldsymbol{Y}_{h}^{*}\right)=\left(\boldsymbol{V}_{h}, \boldsymbol{A}_{j}(\boldsymbol{Y}) \partial_{j} \boldsymbol{Y}_{h}^{*}\right)+\left(\partial_{k} \boldsymbol{V}_{h}, \boldsymbol{K}_{k j}(\boldsymbol{Y}) \partial_{j} \boldsymbol{Y}_{h}^{*}\right),
$$

and the terms related to the subscales as

$$
A_{S}\left(\boldsymbol{Y} ; \boldsymbol{V}_{h}, \tilde{\boldsymbol{Y}}^{*}\right)=\left(\boldsymbol{Y}_{h}, \boldsymbol{A}_{j}(\boldsymbol{Y}) \partial_{j} \tilde{\boldsymbol{Y}}^{*}\right)+\left(\partial_{k} \boldsymbol{V}_{h}, \boldsymbol{K}_{k j}(\boldsymbol{Y}) \partial_{j} \tilde{\boldsymbol{Y}}^{*}\right) .
$$

The objective is to approximate the subscales in order to end up with a problem for the finite element scale alone. For this, we start by integrating by parts the two terms in the RHS of (38), so that we obtain

$$
A_{S}\left(\boldsymbol{Y} ; \boldsymbol{V}_{h}, \tilde{\boldsymbol{Y}}^{*}\right)=\sum_{K}\left(\mathcal{L}^{*}\left(\boldsymbol{Y} ; \boldsymbol{V}_{h}\right), \tilde{\boldsymbol{Y}}^{*}\right)_{K}+\sum_{K}\left(n_{j} \boldsymbol{K}_{k j}^{\top}(\boldsymbol{Y}) \partial_{k} \boldsymbol{V}_{h}, \tilde{\boldsymbol{Y}}^{*}\right)_{\partial K}
$$


Here we have introduced the formal adjoint $\mathcal{L}^{*}(\boldsymbol{U} ; \cdot)$ of the operator $\mathcal{L}(\boldsymbol{U} ; \cdot)$, which is

$$
\mathcal{L}^{*}\left(\boldsymbol{Y} ; \boldsymbol{V}_{h}\right)=-\partial_{j}\left(\boldsymbol{A}_{j}^{\top}(\boldsymbol{Y}) \boldsymbol{V}_{h}\right)-\partial_{k}\left(\boldsymbol{K}_{k j}^{\top}(\boldsymbol{Y}) \partial_{j} \boldsymbol{V}_{h}\right) .
$$

Note that $A_{S}\left(\boldsymbol{Y} ; \boldsymbol{V}_{h}, \widetilde{\boldsymbol{Y}}^{*}\right)$ in (39) involves inter-element jump terms when finite element functions are considered. For continuous solution finite element spaces, the convective term jump at the element boundaries is continuous because it is a function of the variables and, therefore, its sum across adjacent element boundaries is zero. Instead, the diffusive term at the element boundaries in (39) contains derivatives of the variables and it is discontinuous even for continuous finite element spaces. However, this inter-element terms can be neglected by supposing that the subscales vanish at the element boundaries. A further explanation on inter-element subscales can be found in [32]. In this work we approximate the derivatives of the first and second terms on the RHS of the previous expression, respectively as

$$
\begin{gathered}
\partial_{j}\left(\boldsymbol{A}_{j}^{\top}(\boldsymbol{Y}) \boldsymbol{V}_{h}\right) \approx \boldsymbol{A}_{j}^{\top}(\boldsymbol{Y}) \partial_{j} \boldsymbol{V}_{h}+\frac{\partial \boldsymbol{A}_{j}^{\top}(\boldsymbol{Y})}{\partial \boldsymbol{Y}} \partial_{j} \boldsymbol{Y}_{h} \boldsymbol{V}_{h}, \\
\partial_{j}\left(\boldsymbol{K}_{k j}^{\top}(\boldsymbol{Y}) \partial_{k} \boldsymbol{V}_{h}\right) \approx \boldsymbol{K}_{k j}^{\top}(\boldsymbol{Y}) \partial_{j} \partial_{k} \boldsymbol{V}_{h}+\frac{\partial \boldsymbol{K}_{k j}^{\top}(\boldsymbol{Y})}{\partial \boldsymbol{Y}} \partial_{j} \boldsymbol{Y}_{h} \partial_{k} \boldsymbol{V}_{h} .
\end{gathered}
$$

This approximation considers $\partial_{j} \boldsymbol{Y} \approx \partial_{j} \boldsymbol{Y}_{h}$ for the derivatives of the continuous unknowns.

\subsubsection{Subscale equation}

If $\widetilde{\boldsymbol{P}}$ denotes the $L^{2}$ projection onto the space of subscales, it is readily seen that, after integrating by parts the second term of the LHS of (35), the equation for the subscales can be written as

$$
\widetilde{\boldsymbol{P}}\left[\boldsymbol{A}_{0}(\boldsymbol{Y}) \partial_{t} \widetilde{\boldsymbol{Y}}^{*}+\mathcal{L}\left(\boldsymbol{Y} ; \tilde{\boldsymbol{Y}}^{*}\right)\right]=\widetilde{\boldsymbol{P}}\left[\boldsymbol{R}\left(\boldsymbol{Y} ; \boldsymbol{Y}_{h}^{*}\right)\right],
$$

where $\boldsymbol{R}(\cdot, \cdot)$ stands for the finite residual, and is formally defined as $\boldsymbol{R}\left(\boldsymbol{Y} ; \boldsymbol{Y}^{*}\right)=\boldsymbol{F}-\boldsymbol{A}_{0}(\boldsymbol{Y}) \partial_{t} \boldsymbol{Y}^{*}-\mathcal{L}\left(\boldsymbol{Y} ; \boldsymbol{Y}^{*}\right)$. Since the subscales cannot be represented by the finite element mesh, the effect of the nonlinear operator applied to the subscales needs to be approximated. For this, we adopt a diagonal matrix of stabilization parameters that depends on the unknowns $\boldsymbol{\tau}(\boldsymbol{Y})$, such that an approximation of the nonlinear operator applied to the subscales in each element of the form $\mathcal{L}\left(\boldsymbol{Y} ; \tilde{\boldsymbol{Y}}^{*}\right) \approx \boldsymbol{\tau}^{-1}(\boldsymbol{Y}) \widetilde{\boldsymbol{Y}}^{*}$ is made. Hence, for an adequate definition of the projection onto the subscale space, the subscale equation becomes

$$
\widetilde{\boldsymbol{P}}\left[\boldsymbol{A}_{0}(\boldsymbol{Y}) \partial_{t} \widetilde{\boldsymbol{Y}}^{*}+\boldsymbol{\tau}^{-1}(\boldsymbol{Y}) \widetilde{\boldsymbol{Y}}^{*}\right]=\widetilde{\boldsymbol{P}}\left[\boldsymbol{R}\left(\boldsymbol{Y} ; \boldsymbol{Y}_{h}^{*}\right)\right] .
$$

The previous equation is a nonlinear ordinary differential equation, which must be solved at the integration points. Here we use two possibilities to construct the space where the subscales belong. The first and the most common choice is to take it equal to the space of the finite element residuals. That is, to define the projection onto the subscale space as the identity $\widetilde{\boldsymbol{P}}=\boldsymbol{I}$ onto the space of finite element residuals. We call this type of subscales as algebraic subscales (ASGS). The second possibility is the so called orthogonal subscales method (OSGS), which defines the subscales orthogonal to the finite element space, $\widetilde{\boldsymbol{W}}=\boldsymbol{W}_{h}^{\perp}$. In this case, the projection is defined to be the orthogonal projection onto the finite element space $\widetilde{\boldsymbol{P}}=\boldsymbol{P}_{h}^{\perp}=\boldsymbol{I}-\boldsymbol{P}_{h}$, being $\boldsymbol{P}_{h}$ the $L^{2}$-projection onto the finite element space.

Apart from the construction of the spaces where the subscales belong, we call the subscales dynamic if the temporal derivative of the subscales is taken into account. Instead, if this temporal derivative is neglected we call the subscales quasi-static. Besides that, another possibility is to neglect the subscale effect in all the non-linear terms, for example, in the Jacobian and diffusivity matrices, whereas, if we take it into account we call the subscales non-linear. Further results and discussion about these definitions of the subscales may be found in articles such as [33-36] applied to other flow problems.

\subsection{The matrix $\boldsymbol{\tau}$ of stabilization parameters}

The usual compressible definition for the $\tau$ matrix (see [22] for a complete demonstration), includes the local sound velocity that arises from the linearized characteristic compressible flow problem. At the low Mach number limit the sound speed tends to infinity $(c \rightarrow \infty)$, and therefore, that stabilization matrix definition is not suitable. Some authors (e.g. [10,11]) tried to design $\boldsymbol{\tau}$ matrices that were suitable for both the low Mach number limit and high Mach number flows. A necessary requirement for those matrices is the a priori knowledge of numerical parameters 


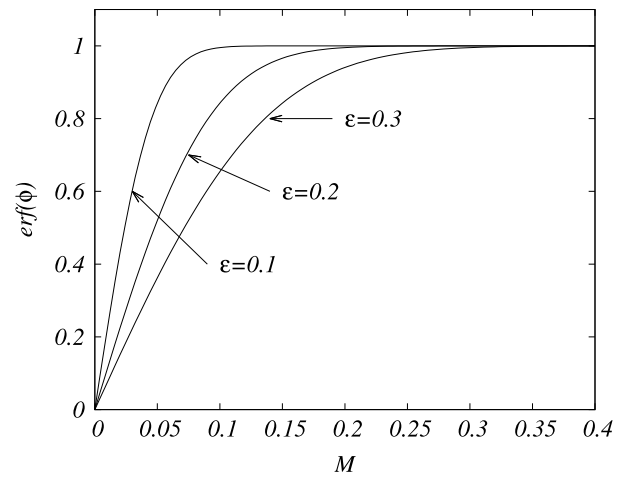

Fig. 1. Gauss error function calculated for different values of parameter $\epsilon$.

depending on the compressibility of the flow. In this sense, we propose the following stabilization matrix for multiple dimensions:

$$
\boldsymbol{\tau}^{-1}(\boldsymbol{Y})=\left[\begin{array}{ccc}
\tau_{c}^{-1}(\boldsymbol{Y}) & \mathbf{0}^{\top} & 0 \\
\mathbf{0} & \tau_{m}^{-1}(\boldsymbol{Y}) \boldsymbol{I} & \mathbf{0} \\
0 & \mathbf{0}^{\top} & \tau_{e}^{-1}(\boldsymbol{Y})
\end{array}\right],
$$

where each component is defined as

$$
\begin{aligned}
\tau_{c}^{-1}(\boldsymbol{Y}) & =\frac{\rho \tau_{m}}{h^{2}}, \\
\tau_{m}^{-1}(\boldsymbol{Y}) & =\frac{C_{1} \mu}{h^{2}}+\frac{C_{2} \rho u^{\star}}{h}, \\
\tau_{e}^{-1}(\boldsymbol{Y}) & =\frac{C_{1} \lambda}{h^{2}}+\frac{C_{2} \rho c_{v} u^{\star}}{h} .
\end{aligned}
$$

In these expressions $C_{1}$ and $C_{2}$ are algorithmic parameters that we take as $C_{1}=12 p^{4}$ and $C_{2}=2 p$, where $p$ is here the order of the finite element interpolation (not the pressure). In the case of the largest characteristic velocity in the convective contribution $(|\boldsymbol{u}|+c)$, instead of using the common definition, we introduce a modified velocity $u^{\star}$, that is calculated with the Gauss error function (commonly denoted as erf),

$$
u^{\star}=|\boldsymbol{u}|+\operatorname{erf}(\phi) c
$$

where $\phi$ is defined as $\phi=2-2(\epsilon-\mathrm{M}) / \epsilon$. Here $\epsilon$ is an algorithmic parameter that determines a certain transition from the compressible to the incompressible regime, which we take as $\epsilon=0.1$ in the numerical examples presented in this work. This definition for the characteristic velocity includes the sound speed, and it converges rapidly to the incompressible definition at the zero Mach limit as shown in Fig. 1. The non-linearity of $\boldsymbol{\tau}(\boldsymbol{Y})$, depending on the velocity and the acoustic speed, is also considered for the subscale equation.

\subsection{Time integration method}

At this point we have described the space discrete stabilized finite element formulation. Let us now comment how we discretize in time.

We partition the time interval $\left(0, t_{f}\right)$ in a sequence of discrete time steps $0=t^{0}<t^{1}<\cdots<t^{N}=t_{f}$, with $\delta t>0$ the time step size, being $t^{n+1}=t^{n}+\delta t$ for $n=0,1,2, \ldots, N$.

In this particular application of compressible flows, more precisely, at low Mach number flows, the acoustic speed tends to infinity. This restricts explicit time stepping schemes to very small time step sizes. Therefore, we avoid this restriction by using an implicit monolithic time integration scheme in order to integrate the time derivatives of Eqs. (34) and (35). More specifically, we use the Backward Differentiation Formula (BDF) scheme. For the time 
dependent function $y(t)$, the BDF approximation of order $k=1,2, \ldots$, is given by $\delta_{k} y^{n+1} / \delta t$, with

$$
\delta_{k} y^{n+1}=\frac{1}{\gamma_{k}}\left(y^{n+1}-\sum_{i=0}^{k-1} \phi_{k}^{i} y^{n-i}\right),
$$

where $\gamma_{k}$ and $\phi_{k}^{i}$ are numerical parameters. In particular, for the first and second order BDF schemes, we have

$$
\begin{aligned}
& \delta_{1} y^{n+1}=y^{n+1}-y^{n}, \\
& \delta_{2} y^{n+1}=\frac{3}{2}\left(y^{n+1}-\frac{4}{3} y^{n}+\frac{1}{3} y^{n-1}\right) .
\end{aligned}
$$

As mentioned before, we use the implicit BDF scheme of first order for discretizing the transient term of the subscales in Eq. (42). That is, we obtain a solution of the subscales at the $n+1$ time step after solving

$$
\widetilde{\boldsymbol{Y}}^{* n+1}=\boldsymbol{\tau}_{t}\left(\boldsymbol{Y}^{n+1}\right)\left(\widetilde{\boldsymbol{P}}\left[\boldsymbol{R}\left(\boldsymbol{Y}^{n+1} ; \boldsymbol{Y}_{h}^{* n+1}\right)\right]+\boldsymbol{A}_{0}\left(\boldsymbol{Y}^{n+1}\right) \frac{\widetilde{\boldsymbol{Y}}^{* n}}{\delta t}\right),
$$

where the dynamic operator $\boldsymbol{\tau}_{t}\left(\boldsymbol{Y}^{n+1}\right)$ is defined as

$$
\boldsymbol{\tau}_{t}\left(\boldsymbol{Y}^{n+1}\right)=\left(\frac{1}{\delta t} \boldsymbol{A}_{0}\left(\boldsymbol{Y}^{n+1}\right)+\boldsymbol{\tau}^{-1}\left(\boldsymbol{Y}^{n+1}\right)\right)^{-1} .
$$

Because the Jacobian matrix of the transient term is a full matrix, the result of the previous expression would lead to a non-diagonal dynamic operator. Our proposal to avoid off-diagonal terms in the stabilization matrix is to choose a diagonal approximation to $\boldsymbol{\tau}_{t}\left(\boldsymbol{Y}^{n+1}\right)$ as follows:

$$
\boldsymbol{\tau}_{t}\left(\boldsymbol{Y}^{n+1}\right) \approx\left[\begin{array}{ccc}
\left(\frac{1}{p^{n+1}} \frac{\rho^{n+1}}{\delta t}+\tau_{c}^{-1}\right)^{-1} & \mathbf{0}^{\top} & 0 \\
\mathbf{0} & \left(\frac{\rho^{n+1}}{\delta t}+\tau_{m}^{-1}\right)^{-1} \boldsymbol{I} & \mathbf{0} \\
0 & \mathbf{0}^{\top} & \left(\frac{\rho^{n+1} c_{p}}{\delta t}+\tau_{e}^{-1}\right)^{-1}
\end{array}\right] .
$$

From [24], we realize that the previous expression is similar to the dynamic operator used in the VMS formulation of the incompressible Navier-Stokes equations and the energy equation.

In the case of the finite element equation (34), we discretize it in time as follows: given the initial conditions $\boldsymbol{Y}^{* 0}$, and supposing that the subscales at the initial time step are identically zero, for $n=1,2, \ldots$, find $\boldsymbol{Y}_{h}^{* n+1} \in \mathcal{W}_{h}$, such that:

$$
\begin{aligned}
& \left(\boldsymbol{V}_{h}, \boldsymbol{A}_{0}\left(\boldsymbol{Y}^{n+1}\right) \frac{\delta_{k} \boldsymbol{Y}_{h}^{* n+1}}{\delta t}\right)+\left(\boldsymbol{V}_{h}, \boldsymbol{A}_{j}\left(\boldsymbol{Y}^{n+1}\right) \partial_{j} \boldsymbol{Y}_{h}^{* n+1}\right) \\
& +\sum_{K}\left(\boldsymbol{V}_{h},\left(\boldsymbol{I}-\boldsymbol{\tau}^{-1}\left(\boldsymbol{Y}^{n+1}\right) \boldsymbol{\tau}_{t}\left(\boldsymbol{Y}^{n+1}\right)\right) \widetilde{\boldsymbol{P}}\left[\boldsymbol{R}\left(\boldsymbol{Y}^{n+1} ; \boldsymbol{Y}_{h}^{* n+1}\right)\right]\right)_{K} \\
& -\sum_{K}\left(\boldsymbol{V}_{h}, \boldsymbol{\tau}^{-1}\left(\boldsymbol{Y}^{n+1}\right) \boldsymbol{\tau}_{t}\left(\boldsymbol{Y}^{n+1}\right) \boldsymbol{A}_{0}\left(\boldsymbol{Y}^{n+1}\right) \frac{\widetilde{\boldsymbol{Y}}^{* n}}{\delta t}\right)_{K} \\
& +\sum_{K}\left(\mathcal{L}^{*}\left(\boldsymbol{Y}^{n+1} ; \boldsymbol{V}_{h}\right), \boldsymbol{\tau}_{t}\left(\boldsymbol{Y}^{n+1}\right)\left(\widetilde{\boldsymbol{P}}\left[\boldsymbol{R}\left(\boldsymbol{Y}^{n+1} ; \boldsymbol{Y}_{h}^{* n+1}\right)\right]+\boldsymbol{A}_{0}\left(\boldsymbol{Y}^{n+1}\right) \frac{\widetilde{\boldsymbol{Y}}^{* n}}{\delta t}\right)\right)_{K} \\
& +\left(\partial_{k} \boldsymbol{V}_{h}, \boldsymbol{K}_{k j}\left(\boldsymbol{Y}^{n+1}\right) \partial_{j} \boldsymbol{Y}_{h}^{* n+1}\right)=\left(\boldsymbol{V}_{h}, \boldsymbol{F}\right) \quad \forall \boldsymbol{V}_{h} \in \mathcal{W}_{h}^{0} .
\end{aligned}
$$

The previous equation, together with Eq. (48), defines the spatial and temporal VMS discretization of the compressible Navier-Stokes equations.

\subsection{Weak imposition of the non-reflecting boundary conditions}

Including the non-reflecting boundary equations (28) into the discrete VMS formulation of the compressible problem (50) is a challenging topic: an explicit solution of the boundary problem requires to fulfill a time step size restriction for stability that is excessive in the case of low Mach number flows. In contrast, it becomes necessary to write the non-reflecting boundary equations in terms of the compressible Navier-Stokes problem and to incorporate 
them in the boundary terms of the variational formulation (via the diffusive fluxes across the domain boundaries) if one aims to solve them fully implicitly.

In order to overcome this difficulty, we prescribe an implicit solution of the non-reflecting boundary conditions in a weak sense by means of introducing some penalization terms to (50). The implicit solution of the non-reflecting boundary conditions can even exploit the time advancing scheme that is already implemented in the stabilized compressible formulation. That is, given the finite element discretization of the boundary problem, we discretize in time the transient term of the characteristic wave equation by using the implicit BDF method described before in this section for the compressible flow problem. In this sense, the same accuracy of the interior domain equations is used to annihilate the propagation signal.

For some positive parameter $\eta>0$, the penalty term containing the non-reflecting equations over the boundary that we add to the LHS of the stabilized implicit formulation is

$$
\eta\left(\boldsymbol{V}_{h}, \boldsymbol{A}_{0}\left(\boldsymbol{Y}^{n+1}\right)\left(\frac{\delta_{k}}{\delta t} \boldsymbol{Y}_{h}^{*, n+1}+\boldsymbol{T}^{-1} \mathcal{L}_{B}\left(\boldsymbol{T} \boldsymbol{Y}_{h}^{n+1} ; \boldsymbol{T} \boldsymbol{Y}_{h}^{*, n+1}\right)\right)\right)_{\Gamma} .
$$

In the same way, the RHS of the stabilized formulation must include the penalty term

$$
\eta\left(\boldsymbol{V}_{h}, \boldsymbol{A}_{0}\left(\boldsymbol{Y}^{n+1}\right)\left(\boldsymbol{T}^{-1} \boldsymbol{F}_{B}+\boldsymbol{T}^{-1} \boldsymbol{S}_{B}\left(\boldsymbol{T} \boldsymbol{Y}_{h}^{n+1}\right) \widehat{\boldsymbol{J}}^{*}\right)\right)_{\Gamma} .
$$

The parameter $\eta$ is defined numerically as $\eta=h \eta_{0}$; in this way, the boundary integral is dimensionally consistent with the variational problem (50), and we have observed from numerical experimentation that $\eta$ needs to scale as the element size $h$. The value of $\eta_{0}$ is specifically defined for each numerical example.

\subsection{Linearization strategy}

The implicit scheme brings the difficulty of solving the non-linearities of the discrete spatial problem. To treat this issue we implement a linearization strategy that is based on Picard's method. At each step $n+1$, we introduce as a superscript an iteration counter $i$, and given $\boldsymbol{Y}_{h}^{n+1, i}$ and $\widetilde{\boldsymbol{Y}}^{n+1, i}$, we compute the finite element unknowns by considering either $\boldsymbol{Y}^{n+1, i} \approx \boldsymbol{Y}_{h}^{n+1, i}$ for the linear subscales, or $\boldsymbol{Y}^{n+1, i} \approx \boldsymbol{Y}_{h}^{n+1, i}+\widetilde{\boldsymbol{Y}}^{n+1, i}$ for the non-linear tracking of the subscales. We use this value in all non-linear terms of Eq. (50). The loop is iterated until the $L^{2}$ norm of the difference between consecutive finite element solutions is below a given convergence criteria, $\left|\phi_{h}^{n+1, i+1}-\phi_{h}^{n+1, i}\right|<\varepsilon\left|\phi_{h}^{n+1, i+1}\right|$, where $\phi_{h}$ stands for any finite element unknown and $\varepsilon$ is the tolerance.

Introducing a separated iterative nested loop of index $j$ for the non-linear equation of the subscales at each iteration $i+1$ of the finite element loop is another possibility. In this case, the subscale unknowns $\widetilde{\boldsymbol{Y}}^{n+1, j+1}$ in Eq. (48) are solved using the finite element unknowns $\boldsymbol{Y}_{h}^{* n+1, i+1}$, and the subscales resulting from the previous iteration $\widetilde{\boldsymbol{Y}}^{n+1, j}$. In some previous articles (e.g. in [35]) this possibility has improved the convergence of the linearization scheme. Nevertheless, we have evaluated it in the problem we consider now, yielding no substantial difference in the convergence rate for the finite element solution; therefore, it has been discarded in the numerical examples to be presented later in the article.

When orthogonal subscales are accounted for, the orthogonal projection of the residual is approximated as

$$
\boldsymbol{P}^{\perp}\left[\boldsymbol{R}\left(\boldsymbol{Y}^{n+1, i} ; \boldsymbol{Y}_{h}^{* n+1, i+1}\right)\right] \approx \boldsymbol{R}\left(\boldsymbol{Y}^{n+1, i} ; \boldsymbol{Y}_{h}^{* n+1, i+1}\right)-\boldsymbol{P}_{h}\left[\boldsymbol{R}\left(\boldsymbol{Y}^{n+1, i} ; \boldsymbol{Y}_{h}^{* n+1, i}\right)\right]
$$

that is to say, the $L^{2}$-projection onto the finite element space $\boldsymbol{P}_{h}$ is evaluated with the unknowns at the end of the previous iteration. Recall that for algebraic subscales $\widetilde{\boldsymbol{P}}=\boldsymbol{I}$, and projection $\boldsymbol{P}_{h}$ is not required. In our implementation, the finite element projection is computed using a lumped mass matrix.

\section{Numerical examples}

In the first part of this section we evaluate the spatial order of accuracy of the numerical formulation in several low Mach number flow cases at steady-state. The method of manufactured solutions is used for two- and three-dimensional domains. Both linear and quadratic approximations are evaluated for the two-dimensional domain. The differential heated cavity and the flow past a cylinder problems are solved next. In both transient problems we illustrate the performance of the proposed VMS method applied to an unsteady low Mach flow. More precisely, we investigate the temporal behavior of the subscales and address the mesh convergence results for the error against a reference solution. The flow around a cylinder is also used to compare the compressible solution at several low Mach numbers 
with the established incompressible one, and to simulate the acoustic propagation of waves, in what is commonly referred as the Aeolian tones aeroacoustic problem. Special emphasis is given to the non-reflecting conditions ability to minimize the wave reflection from the computational boundaries. In the last part of this section the sound generated by the flow past an open cavity is used to test non-linear interactions between the main structures of the flow and the acoustic part of it. The cavity problem at subsonic condition is an aeroacoustic problem that includes very nonlinear flow characteristics and singular pressure points near the corners of the walls.

In all numerical examples the flow is considered as an ideal gas, with ratio of specific heats $\gamma=1.4$ and physical properties $c_{p}=1.010 \mathrm{~kJ} /(\mathrm{kg} \mathrm{K})$ and $c_{v}=0.718 \mathrm{~kJ} /(\mathrm{kg} \mathrm{K})$. At each time step we solve the non-linearities of Eqs. (48) and (50) by using Picard's scheme. This leads to a monotonically decreasing relative error between consecutive iterations, with the subsequent convergence of the numerical method. At most fifteen iterations are performed, fulfilling the maximum relative numerical tolerance for the $L^{2}$ norm iteration residual of $10^{-10}$. As discussed before, iterative solvers can be used for the solution of the linear system of equations as a result of the change of variables in the formulation. Otherwise, the only plausible way to solve transient problems at low Mach numbers is to implement costly direct solvers. In this article, we use the enhanced BiCGstab algorithm [37], which is already implemented in the PETSc parallel solver library [38]. We find that using this method, together with an additive Schwarz method and a block ILU preconditioning [39], greatly improves the convergence and the numerical accuracy of the linear solver.

\subsection{Manufactured solutions}

The first numerical example involves steady state compressible flows at low Mach numbers, which are used to quantify the accuracy of the numerical schemes. The method of manufactured solutions has been traditionally used to quantify the numerical error of partial differential equations solvers. The idea is to generate an exact analytical solution a priori, that is substituted into the continuum equations to obtain a forcing term. This forcing term, which satisfies the compressible Navier-Stokes equations exactly, is applied to the discrete solver. In this example, an exact solution of pressure, velocity and temperature is specified in the computational domain. The manufactured solutions are composed of smooth polynomial analytic functions, which are defined to have non-trivial derivatives and no physical meaning. Reference values of pressure and temperature are fixed as $p_{\text {atm }}=10^{5}$, and $T_{\text {atm }}=300$. Dirichlet boundary conditions are fixed over the boundaries of the computational domain using the relative part of the manufactured solutions. In the following we demonstrate the spatial order of accuracy of several two- and threedimensional elements.

\subsubsection{Two dimensions}

The polynomial functions for the two-dimensional manufactured solutions are given as follows:

$$
\begin{aligned}
p^{*} & =x_{1}^{2} x_{2}^{2}\left(x_{1}-1\right)\left(x_{2}-1\right), \\
u_{1} & =2 x_{1}^{2} x_{2}\left(x_{1}-1\right)^{2}\left(x_{2}-1\right)\left(2 x_{2}-1\right), \\
u_{2} & =-2 x_{1} x_{2}^{2}\left(x_{1}-1\right)\left(x_{2}-1\right)^{2}\left(2 x_{1}-1\right), \\
T^{*} & =2 x_{1}^{2} x_{2}\left(x_{1}^{2}-1\right)\left(x_{2}-1\right),
\end{aligned}
$$

and the contour plots of these fields are presented in Fig. 2. In this two-dimensional problem, the viscosity is fixed to $\mu=10^{-4} \mathrm{~kg} /(\mathrm{m} \mathrm{s})$, and the thermal conductivity to $\lambda=1.0 \mathrm{~W} /(\mathrm{m} \mathrm{K})$. Therefore, the compressibility regime ranges in the $M=\left(0.0,3.5 \times 10^{-5}\right)$ interval, and the local Reynolds number is below $R e=150$, measured with respect to the domain length.

To illustrate one of the key ingredients of the proposed VMS discretization, which is the stabilization matrix design, Fig. 3 shows the local compressibility measured with the Mach number, and the corresponding modified velocity that is used in the stabilization parameter's calculation. It can be noticed that this velocity tends to zero in the zero Mach limit, but at the same time it converges to the acoustic speed at higher compressibility regions. The Gaussian error function used in the calculation of the modified velocity depends on the normalized compressibility, and allows to include the acoustic speed in the stabilization matrix for the compressible regions of the flow, and to equate the compressible and the incompressible stabilization parameters for the momentum and continuity equations at the zero Mach number limit.

To evaluate the accuracy of the numerical method, a series of refined coarse, medium and fine meshes are used in the calculations. The meshes are composed of $Q_{1}$ and $Q_{2}$ elements distributed in a structured fashion. The characteristic 


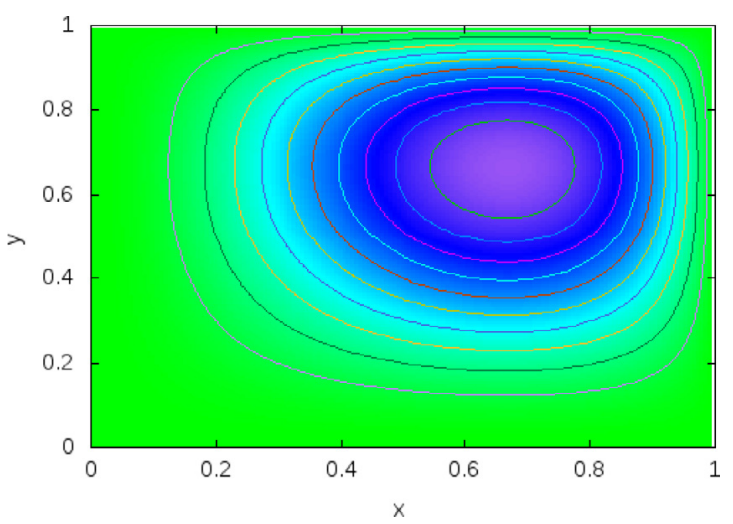

(a) Pressure, $p$.

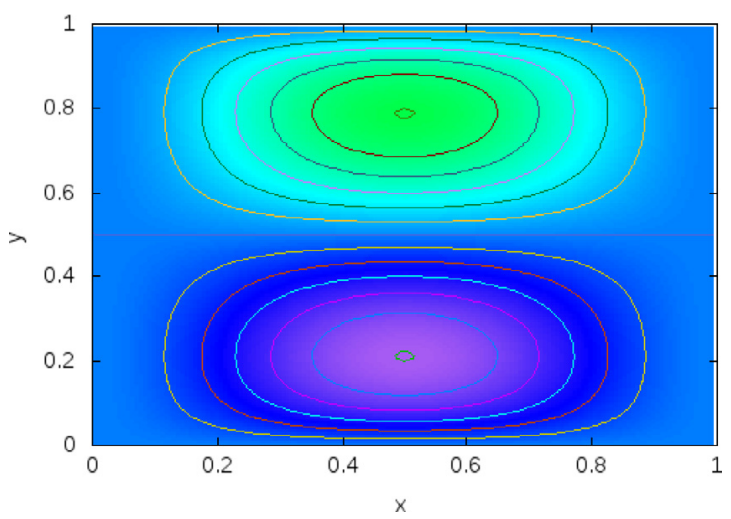

(c) $x_{1}$-velocity.

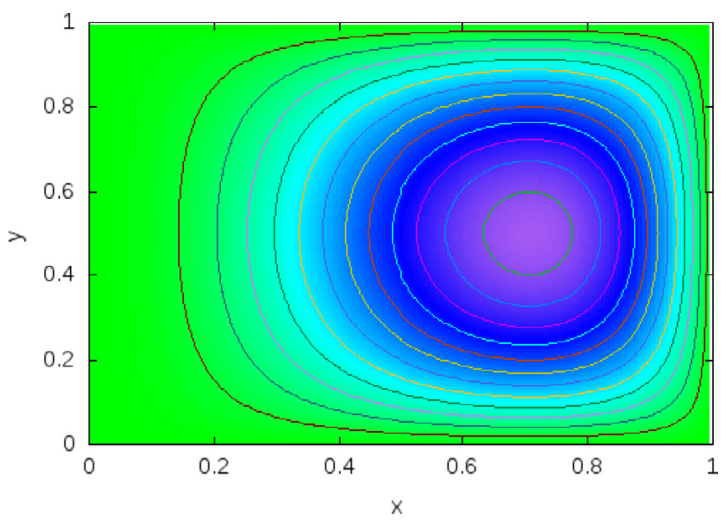

(b) Temperature, $T$.

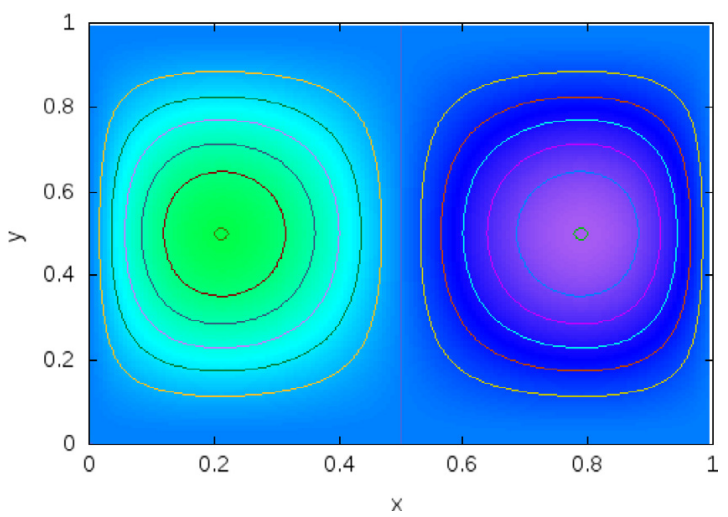

(d) $x_{2}$-velocity.

Fig. 2. Manufactured solutions for the two-dimensional compressible Navier-Stokes equations.

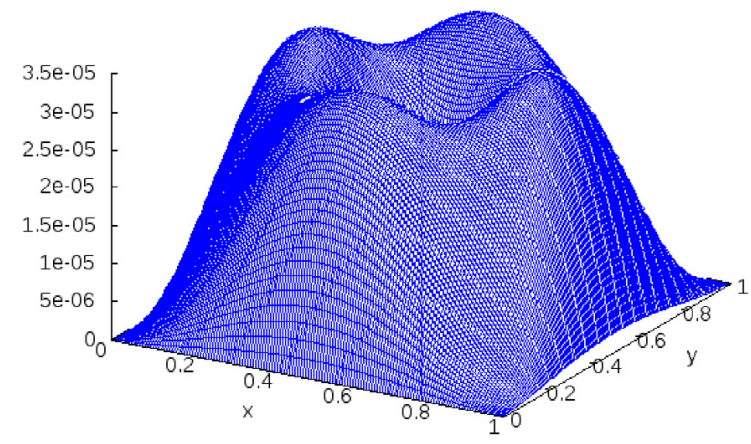

(a) Local Mach number, M.

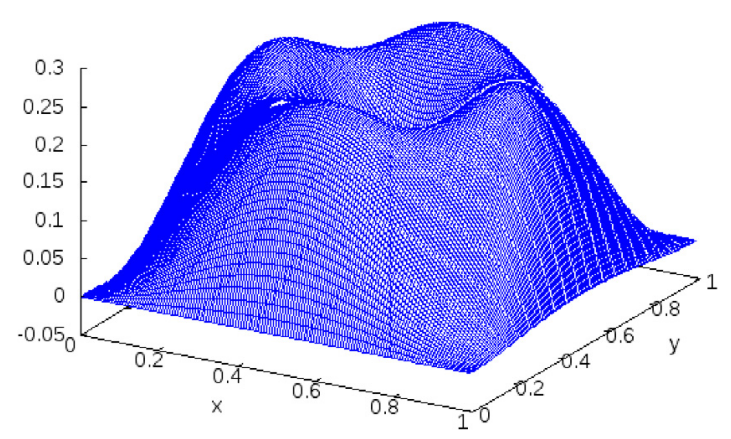

(b) Local sound speed contribution to $u^{\star}, \operatorname{erf}(\phi) c$.

Fig. 3. Characteristic velocity for the two-dimensional compressible Navier-Stokes equations.

element sizes for the $Q_{1}$ meshes are $0.071 \mathrm{~m}, 0.041 \mathrm{~m}$, and $0.022 \mathrm{~m}$, and for the $Q_{2}$ meshes they are $0.1 \mathrm{~m}, 0.05 \mathrm{~m}$, and $0.025 \mathrm{~m}$. The $L^{2}$ norm of the error given between the numerical solution and the exact manufactured solution is used to quantify the accuracy of the computed results.

We first study the results obtained by the VMS discretization comparing the algebraic definition of the subscales, in contrast to the orthogonal subscales. We also include the subscales in the non-linear terms of the variational 


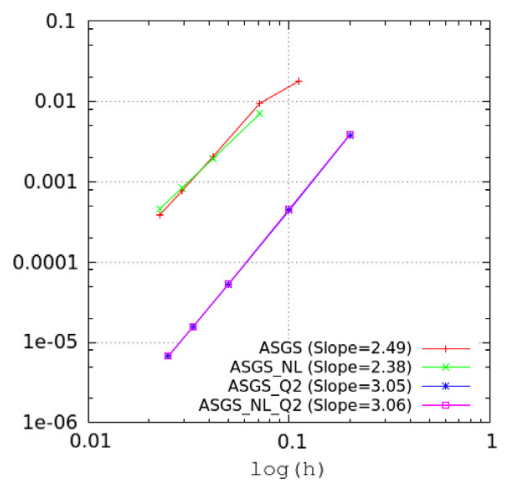

(a) $\left\|p-p_{\text {exact }}\right\|_{2}$.

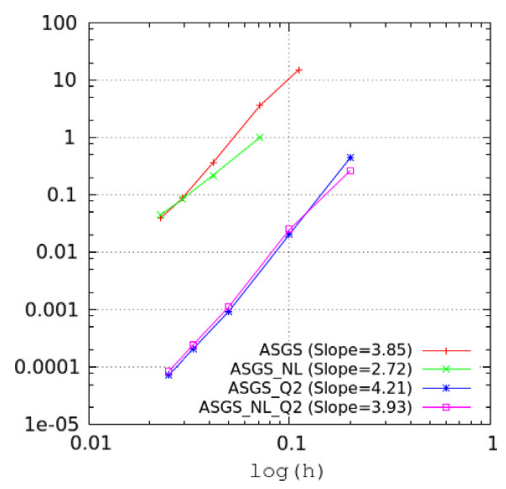

(b) $\left\|T-T_{\text {exact }}\right\|_{2}$.

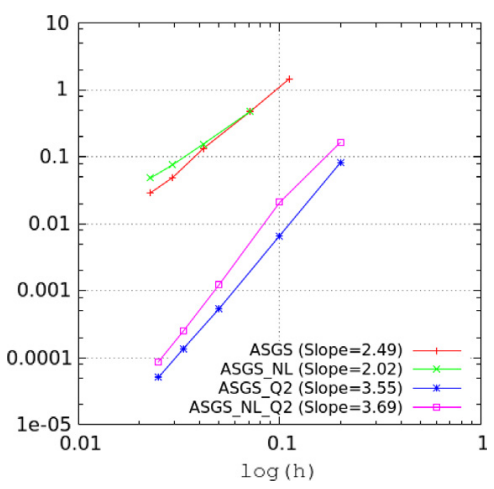

(c) $\left\|u-u_{\text {exact }}\right\|_{2}$.

Fig. 4. Manufactured solutions for the two-dimensional compressible Navier-Stokes equations: $L^{2}$ norm of the exact error defining the space where the subscales live as the finite element residual space (ASGS).

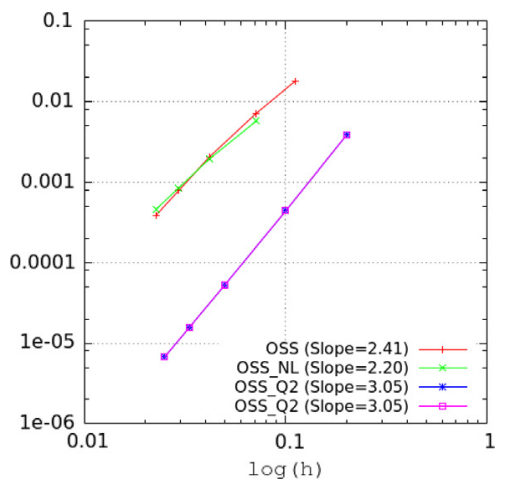

(a) $\left\|p-p_{\text {exact }}\right\|_{2}$.

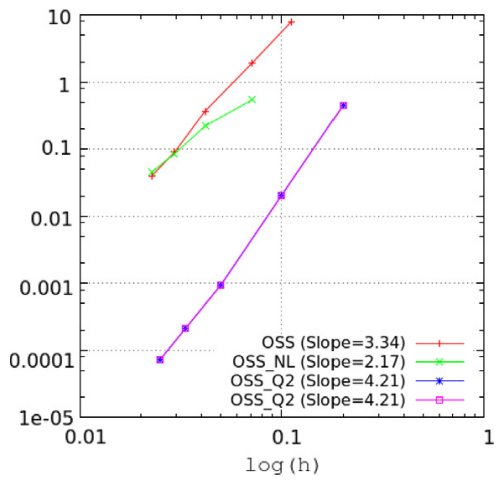

(b) $\left\|T-T_{\text {exact }}\right\|_{2}$.

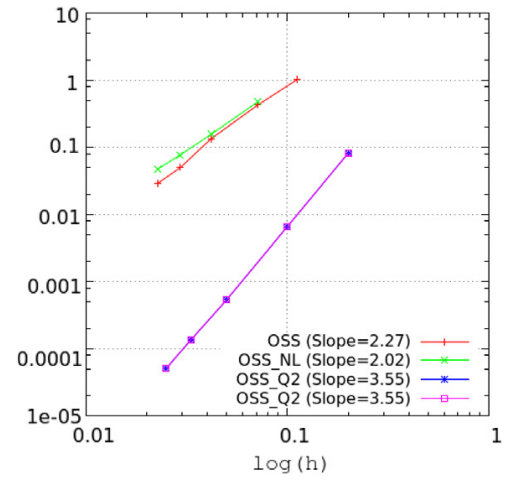

(c) $\left\|u-u_{\text {exact }}\right\|_{2}$

Fig. 5. Manufactured solutions for the two-dimensional compressible Navier-Stokes equations: $L^{2}$ norm of the exact error defining the space where the subscales live as the orthogonal projection to the finite element space (OSGS).

problem (NL). Figs. 4 and 5 depict how the linear and quadratic approximations converge as the mesh is refined. The $L^{2}$ norm of the exact error for pressure, temperature and velocity over the computational domain is presented as a function of the characteristic element sizes. The order of accuracy for the ASGS method, with linear and quadratic approximations, is demonstrated in Fig. 4, whereas, for the OSGS method is presented in Fig. 5. These figures also show the comparison between including the subscales in the non-linear terms and not including them. Results indicate that the order of accuracy is above two for linear elements and above three for quadratic elements. The order of accuracy for temperature and velocity is better than predicted for linear and quadratic approximations, but as predicted for the quadratic approximation of pressure. For the linear approximation, the accuracy given by the fine meshes is comparable to the coarse meshes using the quadratic approximation. In this numerical example it can be observed that the space where the subscales live does not influence the accuracy of the solution. However, including the subscales in the non-linear terms lowers slightly the accuracy and the order of convergence.

\subsubsection{Three dimensions}

Let us consider now the case of three-dimensional computational domains. Similar functions as those used in the previous two-dimensional problem are used. For the pressure, velocity and temperature fields we use, respectively,

$$
\begin{aligned}
& p^{*}=x_{1}^{2} x_{2}^{2} x_{3}^{2}\left(x_{1}-1\right)\left(x_{2}-1\right)\left(1-x_{3}\right), \\
& u_{1}=2 x_{1}^{2} x_{2} x_{3}^{2}\left(x_{1}-1\right)^{2}\left(x_{2}-1\right)\left(2 x_{2}-1\right)\left(1-x_{3}\right),
\end{aligned}
$$




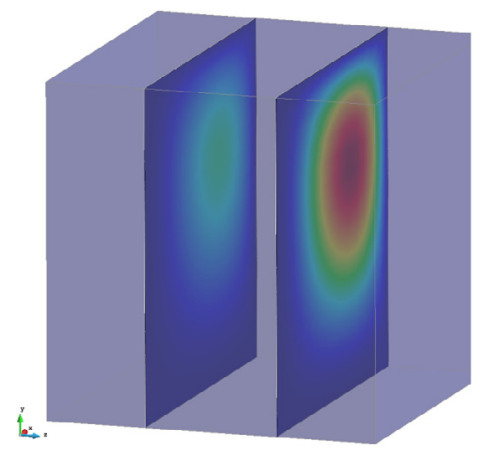

(a) Pressure, $p$.

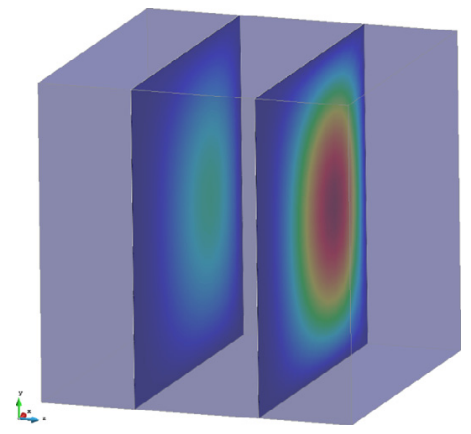

(b) Temperature, $T$.

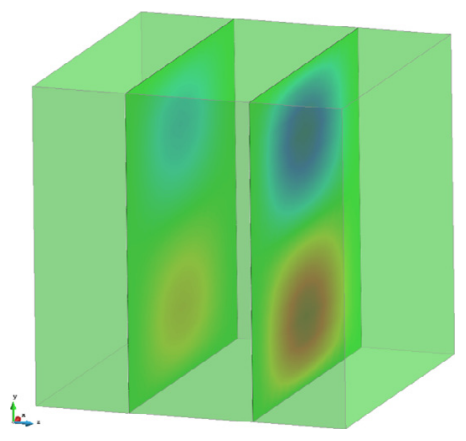

(c) $x_{1}$-velocity.

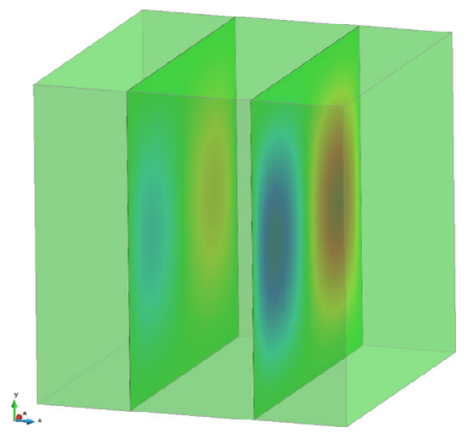

(d) $x_{2}$-velocity.

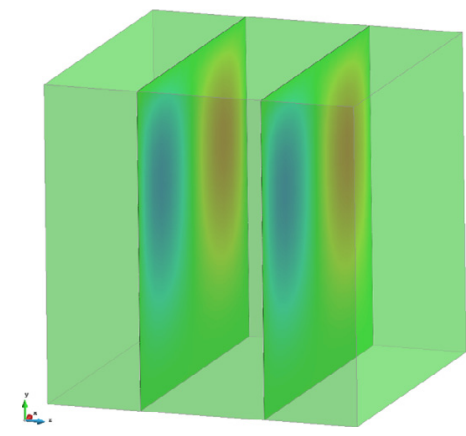

(e) $x_{3}$-velocity.

Fig. 6. Manufactured solutions for the three-dimensional compressible Navier-Stokes equations.

$$
\begin{aligned}
u_{2} & =-2 x_{1} x_{2}^{2} x_{3}^{2}\left(x_{1}-1\right)\left(x_{2}-1\right)^{2}\left(2 x_{1}-1\right)\left(1-x_{3}\right), \\
u_{3} & =-2 x_{1} x_{2}^{2} x_{3}^{2}\left(x_{1}-1\right)\left(2 x_{1}-1\right)\left(x_{2}-1\right)\left(1-x_{3}\right)^{2}, \\
T^{*} & =2 x_{1}^{2} x_{2} x_{3}^{2}\left(x_{1}^{2}-1\right)\left(x_{2}-1\right)\left(1-x_{3}\right) .
\end{aligned}
$$

These three-dimensional fields are presented in Fig. 6 as two-dimensional contours at intersecting planes. In order to preserve the low Mach number condition that has been studied in the two-dimensional problem, the physical properties are fixed to $\mu=10^{-3} \mathrm{~kg} /(\mathrm{m} \mathrm{s})$, and $\lambda=1.0 \mathrm{~W} /(\mathrm{m} \mathrm{K})$. In this case, the order of accuracy of the method is calculated over a sequence of tetrahedral and hexahedral meshes, with $0.052 \mathrm{~m}, 0.034 \mathrm{~m}$, and $0.025 \mathrm{~m}$ of characteristic element sizes. Linear approximations are considered for both types of three-dimensional elements.

Figs. 7 and 8 depict the $L^{2}$ norm of the exact error for pressure, temperature and velocity as a function of the characteristic element sizes. As expected, the observed accuracy is higher for hexahedral than for tetrahedral element meshes. The order of accuracy for both element types is better than predicted for linear approximations. In particular, for the temperature field the order of accuracy is almost five. Contrary to the results of the two-dimensional problem, defining the space where the subscales live as the orthogonal projection to the finite element space, and including the non-linear definition of the subscales, improves the accuracy of the method but lowers the convergence rate.

\subsection{Differentially heated cavity of aspect ratio 8 and $R a=10^{6}$}

The second numerical example is the differentially heated cavity. This is a natural convection flow problem in which the fluid is driven both, by a large temperature gradient between the walls, and by a gravity force. In this numerical example the flow is considered as a two-dimensional problem with the forcing term of the momentum equation playing a significant role in the development of the buoyancy flow patterns. The computational domain is given by a rectangular cavity $[0, L] \times[0, H]$ of aspect ratio $H / L=8$, with $L=1 \mathrm{~m}$. The temperature at the left (hot) wall is fixed to $T_{H}=600 \mathrm{~K}$, and the temperature at the right (cold) wall to $T_{C}=300 \mathrm{~K}$. No slip and 


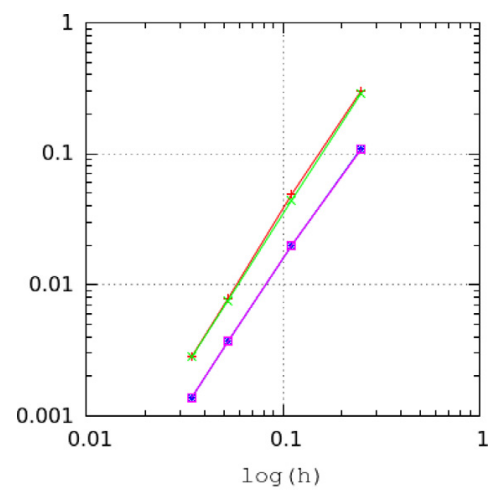

ASGS TETRA (Slope $=2.36$ ) ASGS_NL-TETRA (Slope $=2.34$ ) $\overline{A S G S}$ HEXA (Slope $=2.21)$ ASGS_NL_HEXA (Slope $=2.20)$

(a) $\left\|p-p_{\text {exact }}\right\|_{2}$.

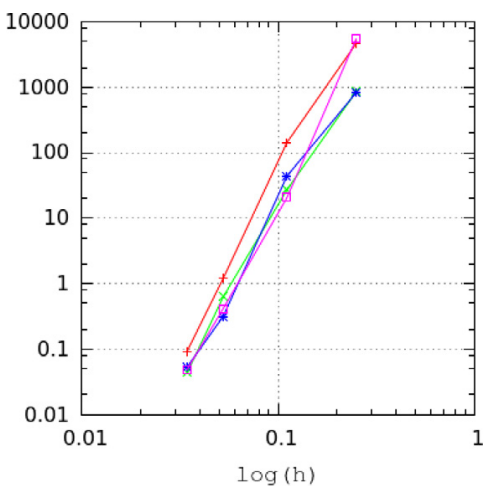

ASGS TETRA (Slope $=5.53$ ) ASGS_NL_TETRA (Slope $=4.95$ ) $\overline{A S G S}$ HEXA (Slope $=5.05) \rightarrow$

(b) $\left\|T-T_{\text {exact }}\right\|_{2}$.

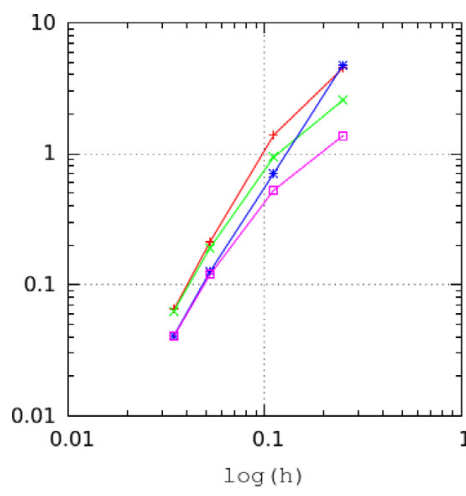

ASGS TETRA (Slope $=2.15$ ) ASGS NL TETRA (Slope $=1.87$ ) $\overline{A S G S}$ HEXA (Slope $=2.39)$ ASGS_NL_HEXA (Slope $=1.77) \longrightarrow$

(c) $\left\|u-u_{\text {exact }}\right\|_{2}$.

Fig. 7. Manufactured solutions for the three-dimensional compressible Navier-Stokes equations: $L^{2}$ norm of the exact error defining the space where the subscales live as the finite element residual space.

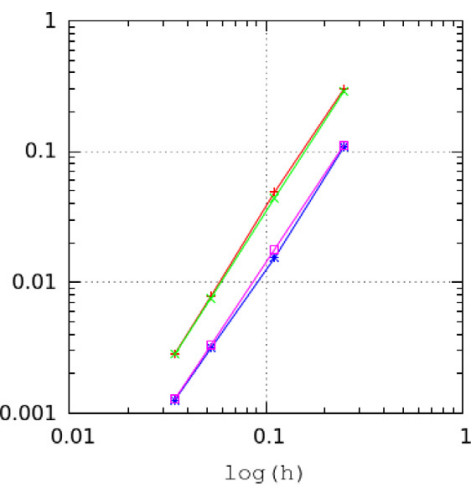

OSS TETRA (Slope $=2.37$ ) OSS_NL TETRA (Slope $=2.34$ ) OSS_HEXA (Slope $=2.25) \longrightarrow \cdots$ OSS_NL_HEXA (Slope $=2.26$ )

(a) $\left\|p-p_{\text {exact }}\right\|_{2}$.

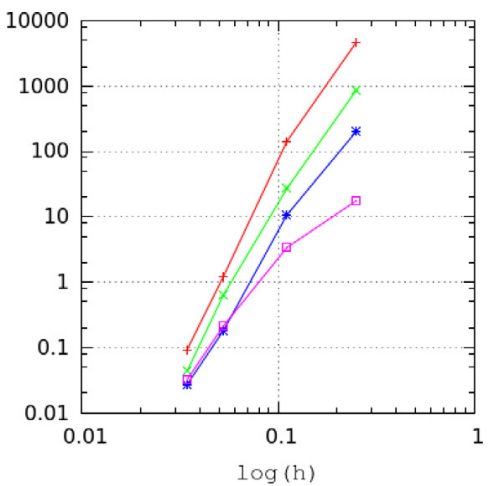

OSS TETRA (Slope $=5.53)$ OSS NL TETRA (Slope $=4.95$ ) OSS̄_HEXA (Slope $=4.59$ ) $\rightarrow$ * OSS_NL_HEXA (Slope $=3.18$ )

(b) $\left\|T-T_{\text {exact }}\right\|_{2}$.

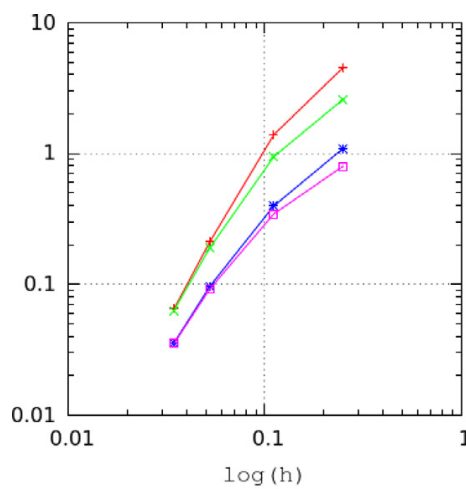

OSS TETRA (Slope $=2.16)$ OSS NL TETRA (Slope $=1.88$ ) OSS_HEXA (Slope $=1.72$ ) $\longrightarrow$ OSS_NL_HEXA (Slope $=1.57) \longrightarrow$

(c) $\left\|u-u_{\text {exact }}\right\|_{2}$.

Fig. 8. Manufactured solutions for the three-dimensional compressible Navier-Stokes equations: $L^{2}$ norm of the exact error defining the space where the subscales live as the orthogonal projection to the finite element space.

impermeable conditions are set over the cavity walls, together with adiabatic boundary conditions for the upper and lower walls. Gravity is specified to be acting in the negative $x_{2}$ direction as $g=(0,-9.8) \mathrm{m} / \mathrm{s}^{2}$. The initial atmospheric pressure $p_{\mathrm{atm}}^{0}=152525 \mathrm{~Pa}$, and the initial temperature of the fluid $T^{0}=450 \mathrm{~K}$, give an initial uniform density of $\rho^{0}=1.16 \mathrm{~kg} / \mathrm{m}^{3}$. The viscosity and thermal conductivity are set to $\mu=2.5 \times 10^{-3} \mathrm{~kg} /(\mathrm{m} \mathrm{s})$, and $\lambda=3.55 \mathrm{~W} /(\mathrm{m} \mathrm{K})$, respectively. The non-dimensional Rayleigh number is defined as the product of the Grashof number and the Prandtl number. In this example, we calculate the Rayleigh number as $R a=|g| \theta \rho^{2} c_{p} /(\mu \lambda)=10^{6}$, where $\theta$ stands for the dimensionless temperature ratio $\theta=2\left(T_{H}-T_{C}\right) /\left(T_{H}+T_{C}\right)=0.66$.

In order to overcome the mechanical restriction of the pressure imposition for transient and variable flows at closed computational domains, an iterative penalization to the mass conservation equation, of the form $\left(q_{h}, \psi\left(p_{h}^{* i+1}-p_{h}^{* i}\right)\right)$ at iteration $i+1$, is included in the stabilized formulation. This penalization guarantees that $p_{h}^{*}$ is solved correctly, up to a constant, when the relative value of pressure is not set at the computational boundary. The factor $\psi$ is selected 


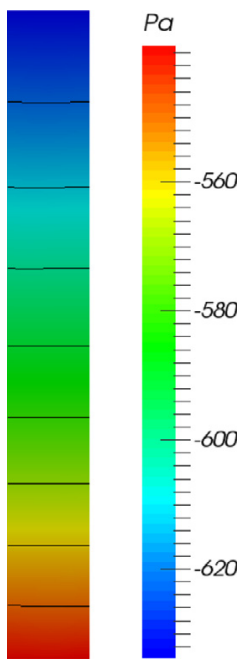

(a) $\overline{p_{h}^{*}}$.

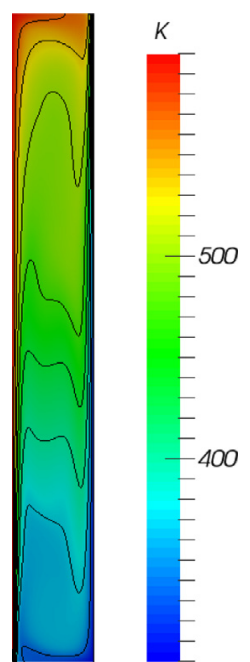

(b) $\overline{T_{h}}$.

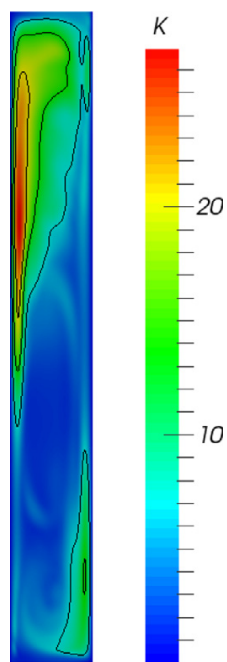

(c) $T_{h, \mathrm{rms}}$.

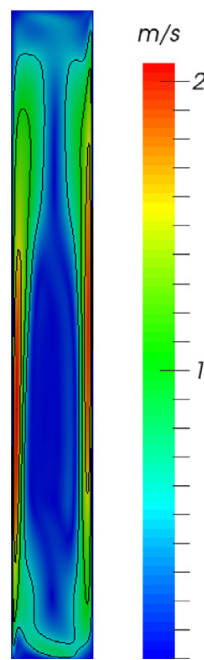

(d) $\left|\overline{\boldsymbol{u}_{h}}\right|$

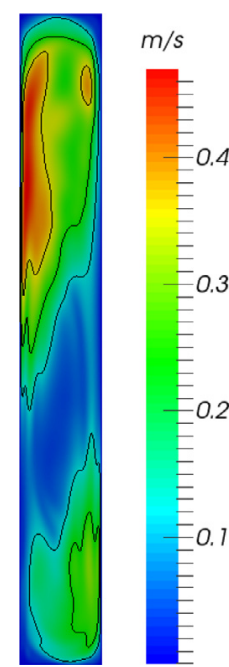

(e) $\left|\boldsymbol{u}_{h, \text { rms }}\right|$.

Fig. 9. Contour results of the differential heated cavity calculated with an ASGS stabilization method and a structured homogeneous mesh containing $Q_{2}$ elements of $h=0.0125 \mathrm{~m}$ size.

numerically as $\psi=10^{-3} \rho / \mu$, in a way that it does not detriment neither the nonlinear scheme convergence (when $\psi$ is large), nor the algebraic solver convergence (when $\psi \rightarrow 0$ ).

In this numerical example we use uniform structured meshes composed of $Q_{2}$ elements. This type of elements helps to provide sensitivity to high order terms of the discrete equations. Specifically, higher order interpolations make possible to include the second order derivatives present in the residual diffusive term and in the adjoint operator, and therefore, to evaluate all terms of the discrete stabilized formulation. We solve three different grid sizes, corresponding to $h_{0}=0.2 \mathrm{~m}, h_{1}=h_{0} / 2 \mathrm{~m}$, and $h_{2}=h_{1} / 2 \mathrm{~m}$. The size of these meshes is defined in terms of two successive divisions $h_{k}=h_{0} / 2^{k}$, where $k=0,1,2$. An additional simulation is performed by solving the problem with the ASGS method and a fine grid of mesh size $h=0.0125 \mathrm{~m}$. This additional solution is used as a reference solution, which allows us to test the accuracy of the VMS stabilization method presented in Section 3. We choose to use the ASGS method as a reference solution because this method is similar to the SUPG method when linear elements are used, and the later has been widely tested in the literature in several compressible flow problems [40]. In all cases, we use the second order accurate BDF as the time integration scheme, with a constant time step size of $\delta t=0.01 \mathrm{~s}$. The simulations are run until the statistically stationary state (measured as the relative error between consecutive transient results of time-averaged variables) is reached.

The reference flow is essentially characterized by an unsteady behavior that is related to the buoyancy produced near the lateral hot and cold walls. The buoyancy is formed as a horizontal shear layer of the velocity component in the direction of gravity, which is in the positive $x_{2}$ direction for the hot wall, and in the negative $x_{2}$ direction for the cold wall. The superposition of the hot and cold wall shear layers generates a broad flow stream cycling around the cavity. The numerical results obtained in the present simulations correspond to the previously referenced solutions in literature, specifically, we obtain similar results to the instant contours of fields presented in [41] for the same cavity ratio and Grashof number. Both the time average and the root mean square values of the reference solution are depicted in Fig. 9. In order to quantify the VMS stabilization attributes, we calculate some statistic measures over the discrete time dependent variables. With regard to the time-dependent discrete solution $\phi_{h}\left(\boldsymbol{x}^{a}, t\right)$ of a certain variable $\phi$ at node $\boldsymbol{x}^{a}$, the time-averaged discrete solution is denoted as $\bar{\phi}_{h}\left(\boldsymbol{x}^{a}\right)$, and the root mean square as $\phi_{h, \mathrm{rms}}\left(\boldsymbol{x}^{a}\right)$. We include the calculation of the root mean square since the time-dependency of the flow is particularly important in this numerical example, and with this calculation we retrieve a measure of the fluctuations strength. In particular, we observe important temperature fluctuations at the top of the hot wall and in the bottom of the cold wall, where the buoyancy reaches its maximum. Root mean square results for temperature also indicate that the hot wall produces most of the fluctuations. The time-averaged temperature results, on the other hand, yield a smooth distribution from the hot 
Table 1

Differential heated cavity error results.

\begin{tabular}{lllll}
\hline & & ASGS & OSGS \\
\cline { 3 - 4 } & & Quasi-static & Quasi-static & Dynamic \\
\hline Error $(\bar{T})$ & $h=0.2$ & $9.26 \times 10^{-3}$ & $9.21 \times 10^{-3}$ & $1.19 \times 10^{-2}$ \\
& $h=0.1$ & $2.05 \times 10^{-3}$ & $2.05 \times 10^{-3}$ & $7.81 \times 10^{-4}$ \\
& $h=0.05$ & $1.05 \times 10^{-4}$ & $1.11 \times 10^{-4}$ & $3.41 \times 10^{-5}$ \\
\hline Error $(|\bar{u}|)$ & $h=0.2$ & 11.2991 & 11.3208 & 8.8726 \\
& $h=0.1$ & 5.127 & 5.1267 & 1.1703 \\
& $h=0.05$ & 2.6753 & 3.7687 & 0.37605 \\
\hline Error $\left(\left|\boldsymbol{u}_{\text {rms }}\right|\right)$ & $h=0.2$ & 7.1079 & 7.237 & 3.3427 \\
& $h=0.1$ & 4.5886 & 0.18367 & 0.31857 \\
& $h=0.05$ & 0.13295 & 0.084087 \\
\hline
\end{tabular}

to the cold wall. We also observe that temperature fluctuations are closely related to fluctuations in the velocity field (expressed by the root mean square values of the velocity magnitude), and that the time-averaged velocity magnitude field exhibits high velocity values near the mid-length of the walls, where the action of the buoyancy is important. On the contrary, the velocity field is almost steady at the center of the cavity, expressed by a small value of both the timeaveraged and root mean square velocity results. On the other hand, the time-averaged pressure field gives an almostlinear distribution along the $x_{2}$ axis, mainly described by the gravity action in that direction. The compressibility range of the simulation is between [0,0.005], measured in terms of the time-averaged non-dimensional Mach number. This compressibility indicates that at any instant of the simulation the flow is subsonic, and even nearly incompressible for certain local regions of the flow.

In order to perform a quantitative comparison between the VMS stabilization methods, we calculate the discrete $L^{2}$ norm of the error by taking

$$
\operatorname{Error}(\bar{\phi})=\frac{\sum_{a}\left(\bar{\phi}_{h}\left(\boldsymbol{x}^{a}\right)-\bar{\phi}\left(\boldsymbol{x}^{a}\right)\right)^{2}}{\sum_{a}\left(\bar{\phi}\left(\boldsymbol{x}^{a}\right)\right)^{2}},
$$

where $\bar{\phi}\left(\boldsymbol{x}^{a}\right)$ refers to the time average of the reference solution at node $\boldsymbol{x}^{a}$. As described before, we consider as the reference solution the one obtained using the ASGS solution and the $h=0.0125 \mathrm{~m}$ mesh. In this numerical example we aim to compare the quasi-static definition for the subscales in contrast to the dynamic subscales. For simplicity, we consider the space where the subscales live as the orthogonal to the finite element space, and the linear definition of the subscales. We also compare with the ASGS quasi-static solution, that can be considered as the traditional VMS method in the literature. The error between coarse grid results and the reference solution is presented in Table 1 for the different mesh sizes. Error calculations are performed for the time-averaged temperature and velocity fields, and for the root mean square of the velocity magnitude. Considering the time-averaged temperature error, this is almost the same for quasi-static subscales in the ASGS and OSGS methods. Instead, the dynamic subscales improve the accuracy for the OSGS method, being considerably more precise by almost an order of magnitude for the smaller mesh sizes. The error of the time-averaged velocity magnitude and the error for the root mean square of the velocity magnitude field are also in line with the error results for temperature.

The mesh convergence results for the error are presented in Fig. 10. Convergence plots are displayed for the error of the time-averaged temperature and velocity magnitude fields. Convergence results for the error of the time-averaged temperature field give a slope greater than three. Oppositely, the convergence order is smaller for the error of the velocity field, both for the time-averaged value and for the root mean square value of the magnitude of velocity; possibly, these results are not inside the asymptotic range. Nevertheless, the most accurate method is the OSGS with the inclusion of the dynamic subscales.

Finally, we present the calculations of the non-dimensional Nusselt number to investigate the transient behavior of the flow field. The Nusselt number relates the heat transferred from the hot to the cold wall, and it is calculated as

$$
\text { Nusselt }(\boldsymbol{x}, t)=\frac{L}{T_{H}-T_{C}} n_{j} \partial_{j} T(\boldsymbol{x}, t), \quad \boldsymbol{x} \in \Gamma, t>0 .
$$




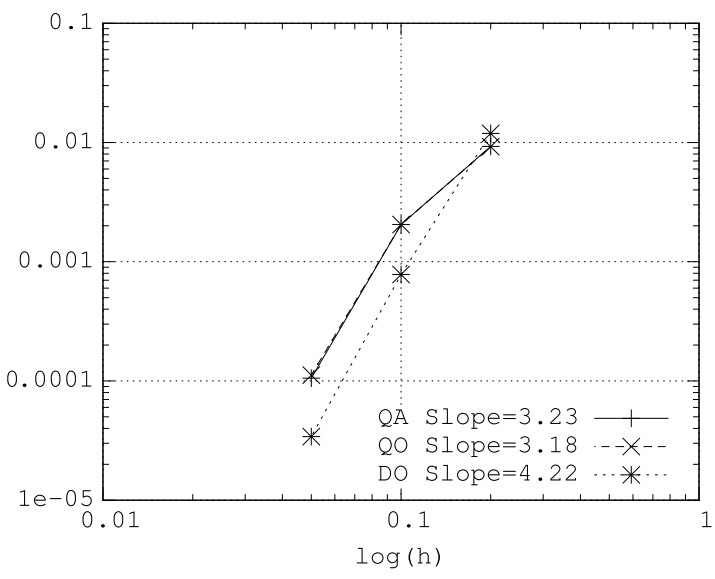

(a) $\bar{T}$.

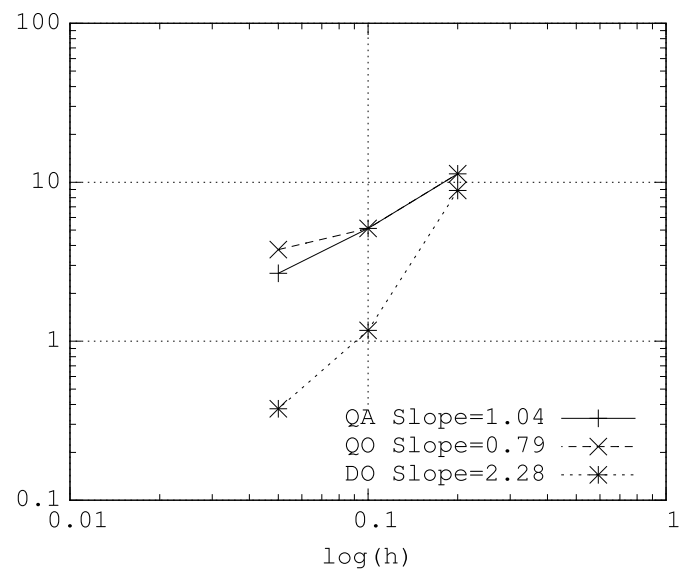

(b) $|\bar{u}|$.

Fig. 10. Convergence results of the differential heated cavity calculated with a structured mesh containing $Q_{2}$ elements of $h=0.0125 \mathrm{~m}$ size: (a) Time-average of temperature, and (b) Time-averaged velocity magnitude.

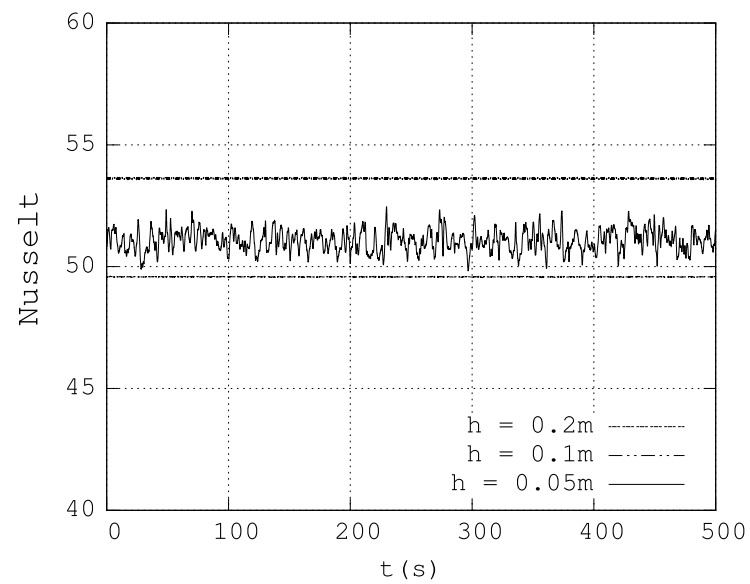

(a) Quasi-static subscales.

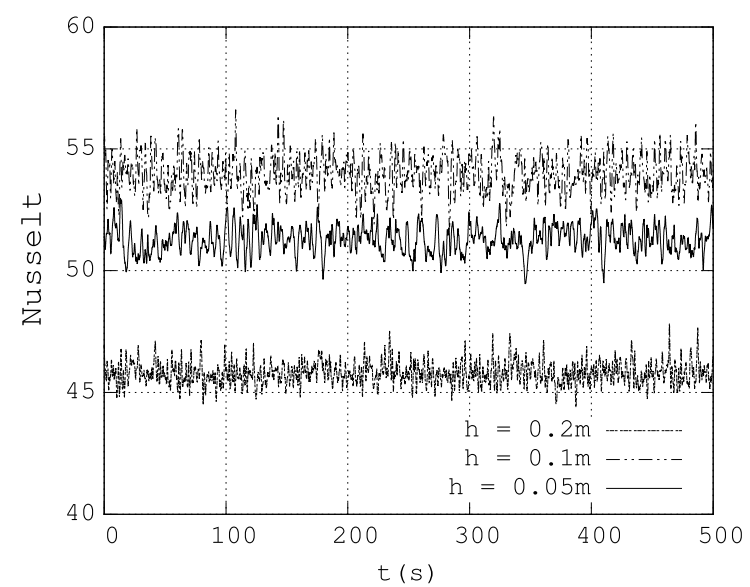

(b) Dynamic subscales.

Fig. 11. Nusselt results of the differential heated cavity calculated with the orthogonal definition of the subscales for three different mesh sizes.

In particular, the transient behavior of the Nusselt number is evaluated by integrating the previous equation over the hot wall. We observe in the transient plots that are displayed in Fig. 11 for the different mesh sizes and stabilizing methods, that the unsteady character of the Nusselt number is subject to the inclusion of the dynamic subscales. This unsteady behavior, which is related to the buoyancy production near the wall, is observed in the reference solution, as well in the $h=0.05 \mathrm{~m}$ mesh size simulations. If we compare the unsteady behavior for the $h=0.2 \mathrm{~m}$ and $h=0.1 \mathrm{~m}$ grids, we observe that the solution given by the dynamic subscales is fluctuating, in contrast to the steady result of the quasi-static subscales. This is, the buoyancy production, and the consequent unsteady behavior of the flow, is sensible to the inclusion of the dynamic subscales. This result is related to the lower error given by the dynamic subscales when compared to the reference solution.

\subsection{Flow past a cylinder}

The third numerical example is the laminar viscous flow past a cylinder. The cylinder is defined to be infinitely long in the axial direction, and immersed in a compressible viscous flow that impinges it uniformly. For a $R e=100$ 


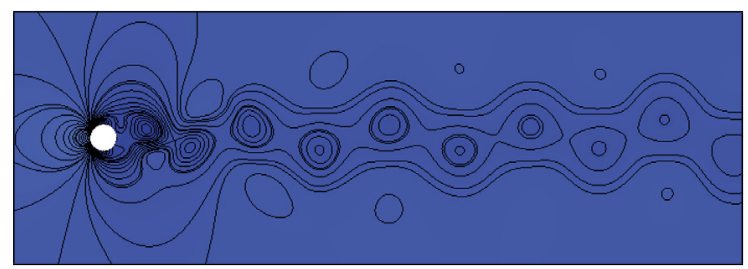

(a)

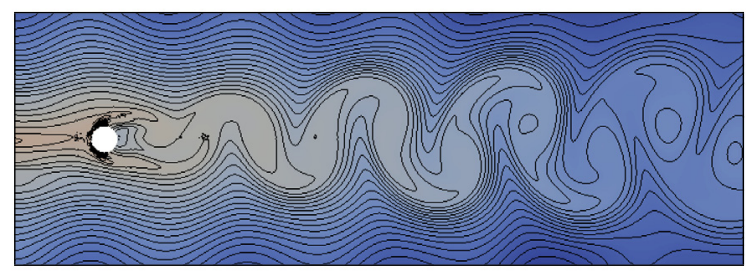

(b)

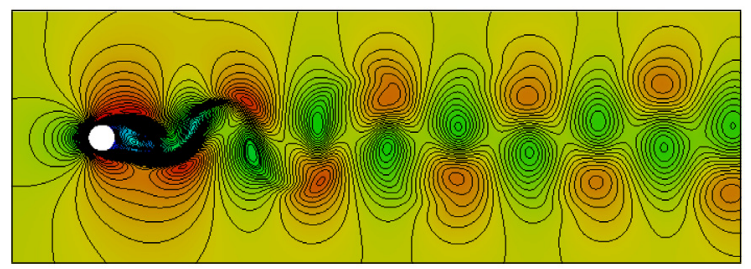

(c)

Fig. 12. Instantaneous contour fields of flow past a cylinder at $M=0.001$ : (a) Pressure, (b) temperature and (c) velocity magnitude calculated with an unstructured mesh containing $P_{1}$ elements of $h=0.001 \mathrm{~m}$ size.

number (based on the cylinder diameter), the flow is unsteady and laminar, developing an oscillating wake behind the cylinder. This example is solved as a two-dimensional problem, and it is a classical unsteady test for viscous incompressible flow solvers which has been used by several authors to quantify the amount of dissipation introduced by a numerical scheme. We use this example to evaluate the performance of the VMS formulation in the low Mach number limit and to investigate its behavior in unsteady flows. The flow around a cylinder is also commonly used to simulate the acoustic propagation of waves. Strong vortices are generated in the wake of the cylinder flow, which are transported downstream and cross the outflow, and cause sound waves due to the perturbation of the pressure field. The waves generated by the vortices behind the cylinder are commonly referred as the Aeolian tones, with a sound wave frequency stable at the fixed value of the wake fluctuation. In the case of the aeroacoustic propagation, special emphasis is given to evaluate the non-reflecting conditions ability to minimize the wave reflection from the computational outlet boundaries.

\subsubsection{Tracking the dynamic subscales}

This first part is intended to further investigate the dynamic subscales behavior in the variational formulation. For doing this, we fix the flow conditions to the free-stream Mach number of $M=0.001$, the Reynolds number to $R e=100$, and the Prandtl number to $\operatorname{Pr}=0.71$. Inlet flow conditions are set by fixing the relative pressure and temperature variables, together with the velocity components. Only the relative pressure needs to be fixed over the outflow boundaries due to the low Mach number condition of the problem. A depiction of the developed flow is presented in Fig. 12. This figure shows the instantaneous pressure, temperature and velocity magnitude contours computed using the present VMS formulation over a fine unstructured mesh of $h=0.001 \mathrm{~m}$ element size and linear triangular elements. The time integration scheme used in the calculation of these results is second order, and the time step size has been kept constant at $\delta t=0.001 \mathrm{~s}$. It can be observed in the figure that, in fact, for this flow conditions, an oscillating wake is developed after the cylinder. Because no qualitative differences can be observed among the results given by the different stabilization methods, we compare them quantitatively by calculating some integral values of the flow, more precisely, we calculate time-integrated values of the lift and drag non-dimensional coefficients. 


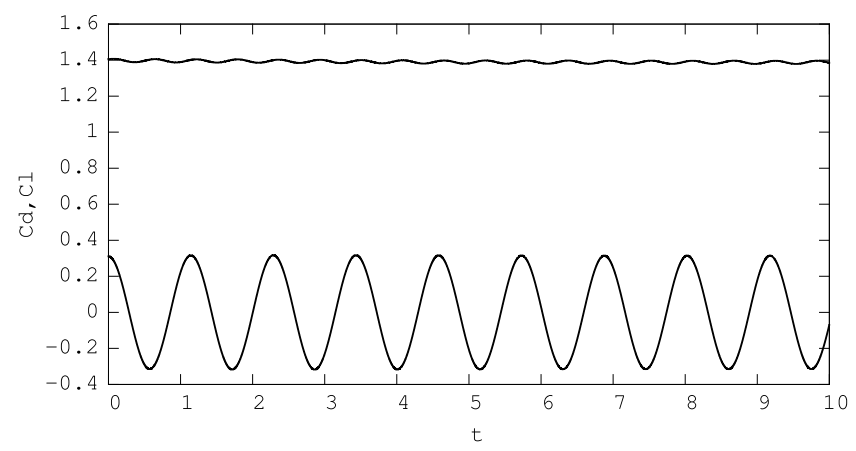

Fig. 13. Time history of drag and lift coefficients for the flow past a cylinder at $M=0.001$. Lift and drag results are calculated with an unstructured mesh containing $P_{1}$ elements of $h=0.001 \mathrm{~m}$ size.

To illustrate this, Fig. 13 shows the time history of the non-dimensional drag and lift coefficients obtained for the previously described flow simulation. Hence, we calculate the time average of $\operatorname{drag} \overline{C_{d}}$, the time $L^{\infty}$ norm of the lift coefficient, and the time $L^{\infty}$ norm of the Strouhal coefficient, over a time-window of 20 s, once the computations have converged to the statistically steady state. For the flow simulation presented in Fig. 12, we obtain the following results: $\overline{C_{d \text { Ref }}}=1.3993,\left|C_{l \text { Ref }}\right|_{\infty}=0.323$, and $\left|\mathrm{St}_{\text {Ref }}\right|_{\infty}=0.17498$. These results agree with the experimental values from the literature, such as those published in [40] for the same free stream conditions, and serve as a reference solution henceforth.

The order of accuracy and the mesh convergence of the stabilization methods is demonstrated by comparing the obtained numerical results and the reference values described before. The meshes that are used to calculate the order of accuracy are also generated in terms of two successive divisions, so that, computations are performed over three different $P_{1}$ unstructured meshes composed of $h_{0}=0.1 \mathrm{~m}$ and 3874 elements, $h_{1}=0.05 \mathrm{~m}$ and 14008 elements, and $h_{2}=0.025 \mathrm{~m}$ and 54884 elements, respectively. The time integration order is $k=2$ for all cases with a constant time step size of $\delta t=0.1 \mathrm{~s}$.

The calculated non-dimensional coefficients are presented in Table 2 for the different VMS definitions. We compare the results obtained including the dynamic definition of subscales, against the quasi-static subscales, by fixing the space where the subscales live as the orthogonal projection to the finite element space (OSGS). As explained in Section 3.1, the dynamic subscales take into account the temporal derivative of the subscales. It can be observed that the dynamic subscales are the most accurate, which exhibit a higher order of accuracy than the quasi-static subscales. In this sense, it is demonstrated that the approximation of the dynamic stabilization operator defined in Eq. (49) is adequate in the low Mach number condition, stabilizing the numerical approximation and improving the accuracy of the variational method. We also test the difference between the accuracy of the method using algebraic (ASGS) and orthogonal subscales, by intentionally defining the subscales as dynamic. In this case, defining the space where the subscales live as the orthogonal to the finite space yields better results for the drag coefficient, but very similar results for the lift and the Strouhal number. No substantial difference in the results has been obtained when the subscales are taken into account in the nonlinear terms of the equations (values not reported).

\subsubsection{Low Mach number limit}

In this second part of the flow past a cylinder example we compare the results obtained by the compressible stabilized formulation with those obtained by the incompressible one. We address the differences in the solution given by both formulations by decreasing the compressibility of the flow from the subsonic to the nearly incompressible regime. For this, we calculate the difference between the integral non-dimensional coefficients obtained by the compressible flow solver at free-stream Mach numbers of 0.001, 0.01, 0.1, 0.2, and 0.5, and those obtained by the incompressible solver (introduced in [24]). The same VMS method is used for both solvers. More specifically, we define the subscales as algebraic, quasi-static and linear, and use the $h=0.025 \mathrm{~m}$ mesh defined in the previous convergence analysis.

Fig. 14 shows the difference between the non-dimensional coefficients obtained by the incompressible solver and the ones obtained by the compressible formulation as a function of the free-stream Mach number. The difference 
Table 2

Flow past a cylinder at $M=0.001$ results.

\begin{tabular}{|c|c|c|c|c|}
\hline & & \multicolumn{2}{|l|}{ OSGS } & \multirow{2}{*}{$\begin{array}{l}\text { ASGS } \\
\text { Dynamic }\end{array}$} \\
\hline & & Quasi-static & Dynamic & \\
\hline Time average of $C_{d}$ & $h=0.1$ & 1.2185 & 1.2401 & 1.1716 \\
\hline \multirow[t]{2}{*}{$\overline{C_{d \mathrm{Ref}}}=1.3993$} & $h=0.05$ & 1.2488 & 1.2746 & 1.2393 \\
\hline & $h=0.025$ & 1.2933 & 1.2924 & 1.2722 \\
\hline Time $L^{\infty}$ norm of $C_{l}$ & $h=0.1$ & 0.0006 & 0.0170 & 0.0260 \\
\hline \multirow{2}{*}{$\left|C_{l \operatorname{Ref}}\right|_{\infty}=0.323$} & $h=0.05$ & 0.1154 & 0.1258 & 0.1189 \\
\hline & $h=0.025$ & 0.1294 & 0.1344 & 0.1346 \\
\hline Time $L^{\infty}$ norm of St & $h=0.1$ & 0.1250 & 0.1250 & 0.1250 \\
\hline \multirow[t]{2}{*}{$\left|S t_{\operatorname{Ref}}\right|_{\infty}=0.17498$} & $h=0.05$ & 0.1333 & 0.1333 & 0.1333 \\
\hline & $h=0.025$ & 0.1538 & 0.1538 & 0.1538 \\
\hline
\end{tabular}

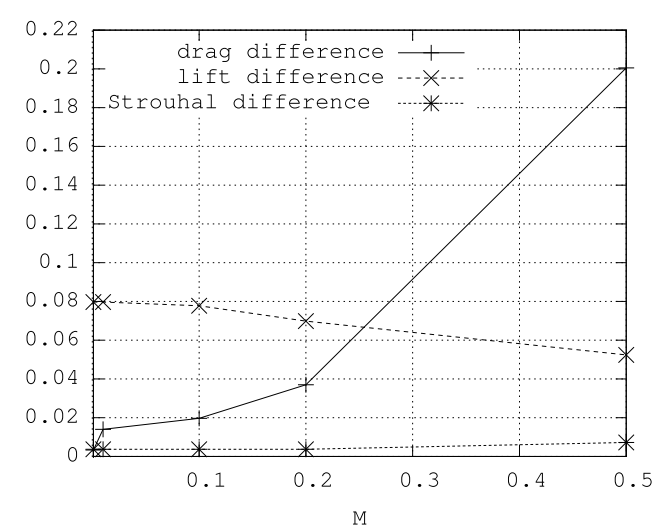

Fig. 14. Flow past a cylinder results: comparison with respect to the incompressible solution.

between the compressible and incompressible solutions with respect to the time averaged drag coefficient scales as the square of the Mach number. It also can be observed that the calculation of the time $L^{\infty}$ norm of the nondimensional lift coefficient is sensible to the mesh definition, and the difference with respect to the incompressible solution decreases with the Mach number. The difference in the Strouhal number is maintained constant for the range of the evaluated Mach numbers.

\subsubsection{Aeolian tones}

The last part of this numerical example demonstrates the ability of the compressible solver to deal with aeroacoustic problems at low Mach number conditions. We exploit the fact that the strong vortices at the wake of the cylinder cause a perturbation of the pressure field which is propagated in the form of sound waves to the far field. We adjust the problem conditions to $R e=1000$ and $M=0.0583$ in order to be able to benchmark the obtained aeroacoustic solution with referenced acoustic simulations. A big portion of the radiated acoustic field is solved with a computational domain that extends 750 times the diameter of the cylinder in each Cartesian direction. The domain is discretized using an unstructured mesh composed of $9293 Q_{2}$ elements, with an element size of $\sim 0.03 D$ at the cylinder surface and $\sim 150 \mathrm{D}$ at the external boundaries.

The minimization of the incident wave reflection at the boundaries is mandatory to avoid spurious waves that affect the wake structure. In this case, a square computational domain is used with the incident waves impinging the boundaries at different oblique angles, so that the non-reflecting boundary conditions are tested. We set the boundary conditions as follows: we fix velocity and temperature for the inlet left-most boundary, while the non-reflecting subsonic boundary condition is solved for the pressure. The non-reflecting subsonic outlet is set for the rest of the external boundaries. For modeling the non-reflecting subsonic outlet with Eq. (29) we fix the pressure relaxation parameter to $\sigma=20$, the wave characteristic length-scale to $l=0.3 D$, and the unperturbed value for pressure 


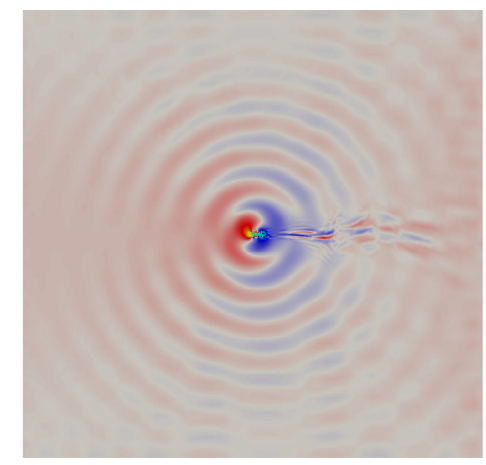

(a)

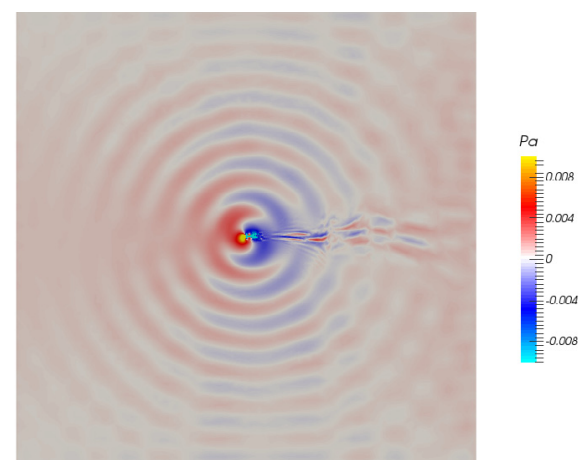

(b)

Fig. 15. Aeolian tones: instantaneous pressure contour in the far field. Two different, (a) and (b), times of the vortex shedding cycle. Relative values of pressure are depicted within a limited range of values.

as $p_{\infty}^{*}=0$. The weak condition (51)-(52) is enforced in every non-reflecting boundary by setting the numerical parameter $\eta=10^{-3}$, for which we have found good convergence of the numerical method.

The implicit solution of the non-reflecting boundaries allows to set a time step size $\delta t=0.01 \mathrm{~s}$, that is only restricted to completely describe the aeroacoustic signal. The simulation is computed using the orthogonal, dynamic and non-linear subscales until the statistical stationary state is reached at about $t=200 \mathrm{~s}$. A depiction of the developed flow is presented in Fig. 15 for two different times of the vortex shedding cycle. In this sense, we accomplish the direct simulation of the acoustic pressure propagation in the Aeolian tones problem using the stabilized compressible formulation. Typically, for far field fluctuations three orders of magnitude smaller than near field fluctuations, the accurate resolution of acoustic fields requires grids containing up to hundred thousands elements. In this case, we can accurately approximate the scattered pressure wave using only 9293 elements in the mesh. Moreover, in our (direct numerical) solution we can observe that the wake structure of the flow is not affected by reflections coming from the artificial computational boundaries, and therefore the non-reflecting boundary conditions exhibit the ability to damp the propagated sound waves and the wake vortices that cross the outflow. In the case of low Mach number flows the characteristic wave approach leads to indistinguishable pressure reflections and distortions at the computational boundaries. At the domain corners, where the waves impinge transversely, the wave is annihilated and no spurious instabilities occur. Although the inlet boundary does not completely damp the pressure wave, and some pile-up is present near the boundary, we have observed that this effect is negligible and does not affect the upstream propagation of sound waves. The inclusion of non-reflecting boundary conditions is crucial in this problem, in which reflections at the boundaries develop oscillations and instabilities at the computational boundaries that end affecting the simulation.

The plot of the pressure wave along the positive $x_{2}$ direction is depicted in Fig. 16 for the same instant of the vortex shedding cycle. A reference solution that was reported in [2] is also included in the plot. In order to test the method, we use the same type of elements and element size, ranging from $\sim 3 \times 10^{-3} \mathrm{D}$ near the cylinder surface to $\sim 30 \mathrm{D}$ at the far field, that was reported in the reference solution. Even though the reference sound wave was obtained in that previous work by applying Lighthill's acoustic analogy over the incompressible flow solution, we observe that the acoustic wave propagation corresponds well in both simulations: the frequency and the amplitude of the radiated sound match the vortex shedding, and the dissipation of the wave is in agreement with the reference solution.

\subsection{Flow past an open cavity}

As a final numerical example, the flow past an open cavity problem at high Reynolds number is simulated. This is a challenging aeroacoustic problem that has been solved in [42-44]. The problem definition is an infinitely long rectangular cavity, of aspect ratio two, with length $L=0.0518 \mathrm{~m}$ and depth $D=0.0254 \mathrm{~m}$, that is commonly placed in a Cartesian domain that extends vertically upward from the cavity, and horizontally at each side of the cavity, so that a portion of the radiated acoustic field is solved. The Cartesian distribution of the domain is typically used for 


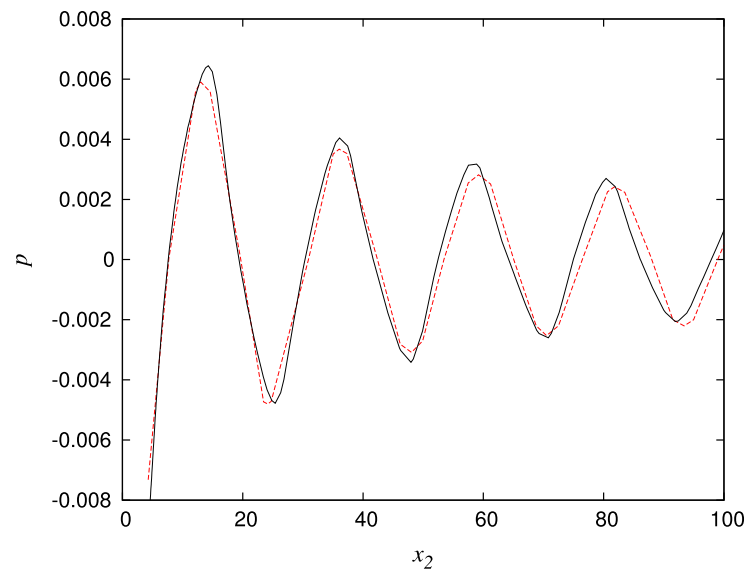

Fig. 16. Aeolian tones: instantaneous pressure along the positive $x_{2}$ direction from the center of the cylinder. Present results are indicated using a solid line. Reference results [2] are denoted with a dashed line.

finite-difference solvers. Instead, our compressible flow solver is capable of using unstructured meshes corresponding to complex geometries, and also to include high order interpolations. In this sense, we solve this problem using a curved far-field boundary, which is sufficiently apart from the cavity, so that a portion of the radiated acoustic field can be resolved. The curvature of the far-field boundary is designed to allow for normal impinging of the scattered sound waves. Setting the origin at the top-center of the cavity, the outer boundary is defined by the circumference that passes through the points $(7 D, 0),(-7 D, 0)$, and $(0,8 D)$. For this problem we consider the $M=0.6$ Mach number condition of the free stream flow, and a $R e=41000$ Reynolds number condition (based on the cavity depth) that has been studied by several authors. We set the sound speed to $c=408 \mathrm{~m} / \mathrm{s}$, the free stream air temperature to $T=408 \mathrm{~K}$, and the pressure to $1.38 \mathrm{~atm}$ in order to accomplish the compressible condition with a free stream velocity of $U=245 \mathrm{~m} / \mathrm{s}$. The Reynolds and Prandtl numbers, on the other hand, impose a free stream density of $\rho=1.16 \mathrm{~kg} / \mathrm{m}^{3}$, a dynamic viscosity of $\mu=1.76 \times 10^{-4} \mathrm{~kg} /(\mathrm{m} \mathrm{s})$, and a thermal conductivity of $\lambda=0.25 \mathrm{~kW} /(\mathrm{m} \mathrm{K})$.

The accurate simulation of the acoustic radiation of the cavity is tightly connected with the definition of the boundaries: because the cavity walls are treated as non slip, impermeable, and isothermal (given by the free stream flow temperature), and as the flow is injected at the left-most side of the curved boundary, a thick boundary layer is generated when the flow passes through the lower non-slip wall. The interaction of the flow disturbances inside the cavity and the upstream boundary layer is the main hydrodynamic feedback mechanism in charge of producing the flow-acoustic interaction. Setting accurately the non-reflecting far field boundaries is crucial to assure that wave reflections do not produce further disturbances to the upstream boundary layer, neither to the flow inside the cavity. The non-reflecting outlet coefficients are set to $\sigma=1$, and $l=2 D$, with the numerical parameter for the weak implicit solution set to $\eta=10^{-2}$. Simulations are performed on the structured mesh depicted in Fig. 17 composed of 9520 bilinear quadrilateral elements using the orthogonal, dynamic and non-linear subscales definition for the compressible solver.

The fluctuating character of the flow can be seen in Fig. 18, in which the normalized spectrum of the scaled $x_{2}$-component of velocity at point $(1.57 \mathrm{D}, 0)$ is presented. The frequency in this plot is normalized with respect to the cavity length and the free stream flow velocity. As reported in previous Direct Numerical Simulations (DNS) [42,43], the present simulation of the flow field is characterized by the circular rolling of vorticity inside the cavity and its unstable impingement over the downstream edge of the cavity. This can be observed as the low frequency modes in the spectrum correspond to the large scale vortex frequencies. The development of the vorticity is produced by singular pressure points located at the top corners of the cavity walls (generated by the boundary layer and the steady flow inside the cavity). For the fully developed flow, the flow-acoustic interaction triggers a highly chaotic behavior inside the cavity. Although turbulence is inherently a three-dimensional phenomena, here we refer to the chaotic character of the flow meaning that the flow is unstable and that the flow is composed by very different and active frequencies, as depicted in the spectrum. 


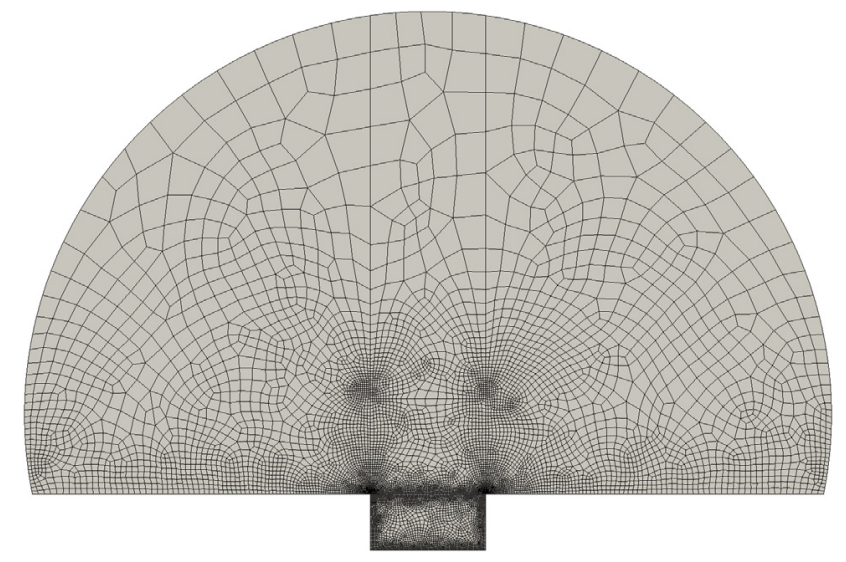

Fig. 17. Flow past an open cavity: Unstructured mesh containing 9520 total $Q_{2}$ elements.

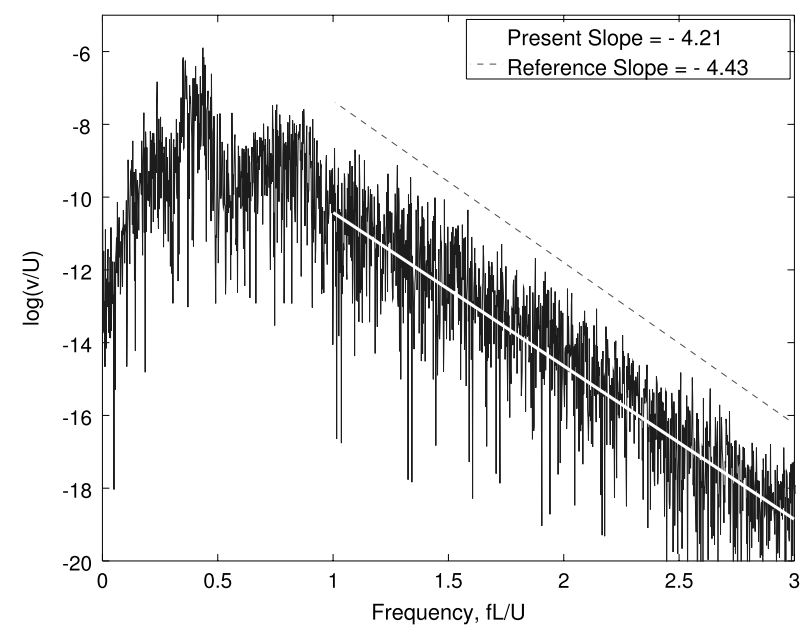

Fig. 18. Flow past an open cavity: normalized spectrum of the scaled $x_{2}$-component of velocity at $(1.57 D, 0)$.

The spectrum of Fig. 18 is also characterized by a cascade that is similar to that encountered in fluid flow turbulence, containing a range that behaves as $(f L / U)^{-4.2}$. This decay corresponds with the one obtained by the DNS solution in [42] using a sixth-order accurate finite difference solver with half a million grid points. The two lower peaks are also reported in [42]: one at around $f L / U=0.4$, and another at $f L / U=0.7$. In this sense, we observe that low frequency modes correspond well in both simulations to the vortex shedding frequencies, and that the present simulation is able to represent the scales of sound using a grid that is two orders of magnitude smaller than the benchmark simulation. The frequency peak reported by [45] for the acoustic radiation measurement using an experimental setting for the same problem definition is $f L / U=0.8$, which is higher than the present simulation results mainly due to threedimensional effects.

Scattering of acoustic waves can be visualized and compared in Fig. 19. In this figure we depict the instantaneous pressure contour obtained in the present simulation, in contrast to the photograph from [45] (depicted at the left side of the figure) showing the structure of the radiated field. There is a qualitative agreement between the present simulation and the experimental setting: visual comparisons of the scattered pressure waves coincide. The sound generated in those pressure waves is involved with the mixing and the non-linear interaction between the very different scales, and consequently, for mesh sizes that cannot resolve all the flow scales (such as the mesh of Fig. 17), the production of sound demonstrates a strong sensitivity to the amount of diffusion given by the numerical method [46]. In this sense, the accurate definition of the sound waves given in the present simulation relies only on the numerical diffusion 


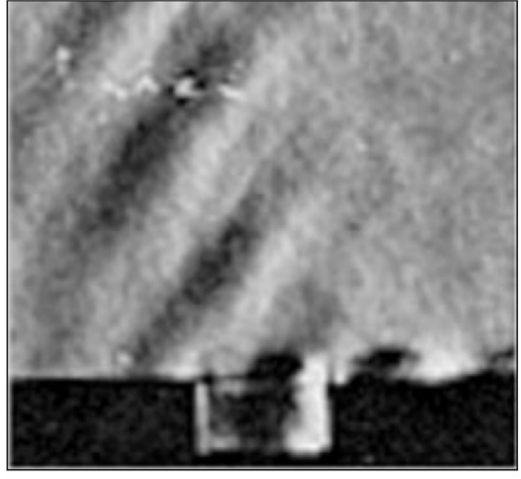

(a)

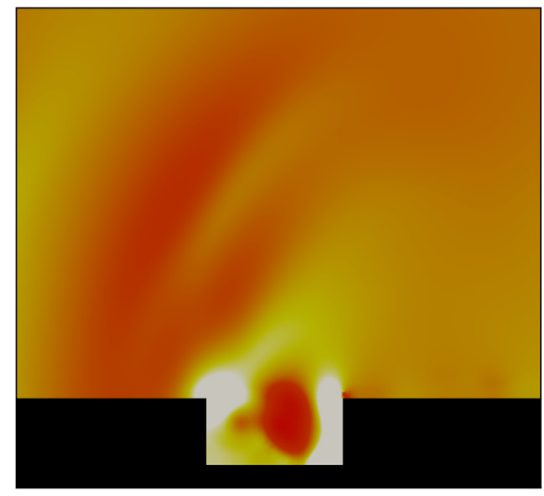

(b)

Fig. 19. Flow past an open cavity comparison: (a) Schlieren photographs [45] at $\mathrm{M}=0.64$, (b) instantaneous contour of the pressure field at $\mathrm{M}=0.6$.

given the VMS stabilization method, without any modification of the continuous problem or the inclusion of any turbulence model for the subgrid scales. This kind of approximation has been recently related to the Implicit LES (ILES) methods [47], which accurately represent the underlying turbulent behavior by the addition of dissipative numerical terms solely, even if the mesh is not too fine to resolve the majority of the flow scales.

It is also important to note that the curved far field boundary does not affect the solution as it damps completely the reflection of the sound wave. The pressure scattering shown in Fig. 19 is mainly a combination of direct and reflected acoustic waves, and this agreement cannot be achieved without the use of non-reflecting boundary conditions (or with the inclusion of some damping technique). Spurious oscillations, that lead to instabilities in the flow, also appear in this highly non-linear aeroacoustic problem if some reflections of waves occur. In this sense, we obtain accurate results for a grid size which is composed only by 9520 elements, by applying an accurate approximation of the compressible Navier-Stokes equations and the numerical strategy for damping wave reflections at the computational boundaries.

\section{Conclusions}

In this article, a finite element approximation of the compressible Navier-Stokes equations written in primitive variables has been developed. From the numerical point of view, the compressible model has been approximated by a stabilized VMS method, and an implicit scheme has been used to advance in time. Some other ingredients, such as the orthogonal, dynamic and non-linear definition of the subscales, and the weak imposition of non-reflecting boundary conditions for simulating aeroacoustic flows, have been investigated. In particular, the design of the static and transient stabilization matrix, and the decomposition of the pressure and temperature unknowns into a relative and a reference part allow to solve nearly incompressible cases.

The accuracy of the method with the mesh size has been verified for two- and three-dimensional linear elements, as well as for two-dimensional quadratic elements, using steady and low Mach manufactured solutions. The differential heated cavity problem and the flow past a cylinder problem have been used to test the accuracy of the method for dynamic cases. It has been observed that including the temporal derivatives of the subscales, and defining the space where the subscales live as the orthogonal to the finite element space, improve the accuracy of the variational method. Finally, the possibility of directly computing the acoustic pressure waves with the compressible NavierStokes equations has been validated with the Aeolian tones problem and with the flow past an open cavity problem. Accurate simulations of acoustic radiation have been obtained by solving directly the compressible Navier-Stokes equations and damping wave reflections at the computational boundaries. The weak imposition of non-reflecting boundary conditions allows for the free propagation of acoustic waves within the implicit time stepping scheme.

\section{Acknowledgments}

C. Bayona acknowledges the doctoral scholarship received from the Colombian Government - Colciencias. J. Baiges acknowledges the support of the Spanish Government through the Ramón y Cajal grant RYC-2015-17367. R. 
Codina acknowledges the support received from the ICREA Acadèmia Program, from the Catalan Government. This work is partially funded through the ELASTIC-FLOW project, Ref. DPI2015-67857-R of the Spanish Government. The authors thankfully acknowledges the computer resources, technical expertise and assistance provided by the Red Española de Supercomputación (RES-BSC).

\section{References}

[1] J. Wong, D. Darmofal, J. Peraire, The solution of the compressible Euler equations at low Mach numbers using a stabilized finite element algorithm, Comput. Methods Appl. Mech. Engrg. 190 (43) (2001) 5719-5737.

[2] O. Guasch, R. Codina, Computational aeroacoustics of viscous low speed flows using subgrid scale finite element methods, J. Comput. Acoust. 17 (03) (2009) 309-330.

[3] J. Principe, R. Codina, Mathematical models for thermally coupled low speed flows, Adv. Theor. Appl. Mech. 2 (2009) $93-112$.

[4] T. Yabe, P.-Y. Wang, Unified numerical procedure for compressible and incompressible fluid, J. Phys. Soc. Japan 60 (7) (1991) $2105-2108$.

[5] O.C. Zienkiewicz, R. Codina, A general algorithm for compressible and incompressible flow-Part I. The split, characteristic-based scheme, Internat. J. Numer. Methods Fluids 20 (8-9) (1995) 869-885.

[6] R. Codina, M. Vázquez, O.C. Zienkiewicz, A general algorithm for compressible and incompressible flows. Part III: The semi-implicit form, Internat. J. Numer. Methods Fluids 27 (1-4) (1998) 13-32.

[7] F. Xiao, R. Akoh, S. Ii, Unified formulation for compressible and incompressible flows by using multi-integrated moments II: Multidimensional version for compressible and incompressible flows, J. Comput. Phys. 213 (1) (2006) 31-56.

[8] L. Pesch, J.J. van der Vegt, A discontinuous Galerkin finite element discretization of the Euler equations for compressible and incompressible fluids, J. Comput. Phys. 227 (11) (2008) 5426-5446.

[9] T.J. Hughes, L. Franca, M. Mallet, A new finite element formulation for computational fluid dynamics: I. Symmetric forms of the compressible Euler and Navier-Stokes equations and the second law of thermodynamics, Comput. Methods Appl. Mech. Engrg. 54 (2) (1986) $223-234$.

[10] G. Hauke, T. Hughes, A comparative study of different sets of variables for solving compressible and incompressible flows, Comput. Methods Appl. Mech. Engrg. 153 (1) (1998) 1-44.

[11] M. Billaud, G. Gallice, B. Nkonga, A simple stabilized finite element method for solving two phase compressible-incompressible interface flows, Comput. Methods Appl. Mech. Engrg. 200 (9) (2011) 1272-1290.

[12] T. Colonius, S.K. Lele, P. Moin, Boundary conditions for direct computation of aerodynamic sound generation, AIAA J. 31 (9) (1993) $1574-1582$.

[13] B. Engquist, A. Majda, Absorbing boundary conditions for numerical simulation of waves, Proc. Natl. Acad. Sci. 74 (5) (1977) $1765-1766$.

[14] D.H. Rudy, J.C. Strikwerda, A nonreflecting outflow boundary condition for subsonic Navier-Stokes calculations, J. Comput. Phys. 36 (1) (1980) 55-70.

[15] A. Bayliss, E. Turkel, Radiation boundary conditions for wave-like equations, Comm. Pure Appl. Math. 33 (6) (1980) $707-725$.

[16] T. Colonius, Modeling artificial boundary conditions for compressible flow, Annu. Rev. Fluid Mech. 36 (2004) $315-345$.

[17] T. Hughes, Multiscale phenomena: Green's functions, the Dirichlet-to-Neumann formulation, subgrid scale models, bubbles and the origins of stabilized methods, Comput. Methods Appl. Mech. Engrg. 127 (1) (1995) 387-401.

[18] T.J. Hughes, G. Scovazzi, T.E. Tezduyar, Stabilized methods for compressible flows, J. Sci. Comput. 43 (3) (2010) $343-368$.

[19] R. Codina, S. Badia, J. Baiges, J. Principe, Variational multiscale methods in computational fluid dynamics, in: E. Stein, R. de Borst, T.J. Hughes (Eds.), Encyclopedia of Computational Mechanics, Wiley Online Library.

[20] M. Polner, L. Pesch, J. Van Der Vegt, Construction of stabilization operators for Galerkin least-squares discretizations of compressible and incompressible flows, Comput. Methods Appl. Mech. Engrg. 196 (21) (2007) 2431-2448.

[21] R. Codina, Finite element approximation of the convection-diffusion equation: Subgrid-scale spaces, local instabilities and anisotropic spacetime discretizations, in: BAIL 2010-Boundary and Interior Layers, Computational and Asymptotic Methods, Springer, 2011, pp. 85-97.

[22] C.A. Bayona Roa, J. Baiges, R. Codina, Variational multi-scale finite element approximation of the compressible Navier-Stokes equations, Internat. J. Numer. Methods Heat Fluid Flow 26 (3/4) (2016) 1240-1271.

[23] R. Codina, Stabilization of incompressibility and convection through orthogonal sub-scales in finite element methods, Comput. Methods Appl. Mech. Engrg. 190 (13) (2000) 1579-1599.

[24] R. Codina, J. Principe, O. Guasch, S. Badia, Time dependent subscales in the stabilized finite element approximation of incompressible flow problems, Comput. Methods Appl. Mech. Engrg. 196 (21) (2007) 2413-2430.

[25] R. Codina, A stabilized finite element method for generalized stationary incompressible flows, Comput. Methods Appl. Mech. Engrg. 190 (20) (2001) 2681-2706.

[26] K.W. Thompson, Time dependent boundary conditions for hyperbolic systems, J. Comput. Phys. 68 (1) (1987) 1-24.

[27] T.J. Poinsot, S. Lele, Boundary conditions for direct simulations of compressible viscous flows, J. Comput. Phys. 101 (1) (1992) $104-129$.

[28] G. Lodato, P. Domingo, L. Vervisch, Three-dimensional boundary conditions for direct and large-eddy simulation of compressible viscous flows, J. Comput. Phys. 227 (10) (2008) 5105-5143.

[29] P. Fosso, H. Deniau, N. Lamarque, T. Poinsot, et al., Comparison of outflow boundary conditions for subsonic aeroacoustic simulations, Internat. J. Numer. Methods Fluids 68 (10) (2012) 1207-1233.

[30] G. Hauke, A. Landaberea, I. Garmendia, J. Canales, A segregated method for compressible flow computation. Part II: General divariant compressible flows, Internat. J. Numer. Methods Fluids 49 (2) (2005) 183-209.

[31] C.K. Tam, Computational aeroacoustics-Issues and methods, AIAA J. 33 (10) (1995) 1788-1796.

[32] R. Codina, J. Principe, J. Baiges, Subscales on the element boundaries in the variational two-scale finite element method, Comput. Methods Appl. Mech. Engrg. 198 (5) (2009) 838-852. 
[33] R. Codina, J. Principe, Dynamic subscales in the finite element approximation of thermally coupled incompressible flows, Internat. J. Numer. Methods Fluids 54 (6-8) (2007) 707-730.

[34] M. Avila, J. Principe, R. Codina, A finite element dynamical nonlinear subscale approximation for the low Mach number flow equations, J. Comput. Phys. 230 (22) (2011) 7988-8009.

[35] M. Avila, R. Codina, J. Principe, Large eddy simulation of low mach number flows using dynamic and orthogonal subgrid scales, Comput. \& Fluids 99 (2014) 44-66.

[36] O. Colomés, S. Badia, R. Codina, J. Principe, Assessment of variational multiscale models for the large eddy simulation of turbulent incompressible flows, Comput. Methods Appl. Mech. Engrg. 285 (2015) 32-63.

[37] H.A. Van der Vorst, Bi-CGSTAB: A fast and smoothly converging variant of Bi-CG for the solution of nonsymmetric linear systems, SIAM J. Sci. Stat. Comput. 13 (2) (1992) 631-644.

[38] S. Balay, S. Abhyankar, M.F. Adams, J. Brown, P. Brune, K. Buschelman, L. Dalcin, V. Eijkhout, W.D. Gropp, D. Kaushik, M.G. Knepley, L.C. McInnes, K. Rupp, B.F. Smith, S. Zampini, H. Zhang, PETSc Web page, 2015. [Online]. Available: http://www.mcs.anl.gov/petsc.

[39] T.F. Chan, H.A. Van der Vorst, Approximate and incomplete factorizations, in: Parallel Numerical Algorithms, Springer, 1997, pp. 167-202.

[40] S. Mittal, T. Tezduyar, A unified finite element formulation for compressible and incompressible flows using augmented conservation variables, Comput. Methods Appl. Mech. Engrg. 161 (3-4) (1998) 229-243.

[41] R. Codina, J. Principe, M. Ávila, Finite element approximation of turbulent thermally coupled incompressible flows with numerical sub-grid scale modelling, Internat. J. Numer. Methods Heat Fluid Flow 20 (5) (2010) 492.

[42] C.W. Rowley, T. Colonius, A.J. Basu, On self-sustained oscillations in two-dimensional compressible flow over rectangular cavities, J. Fluid Mech. 455 (2002) 315-346.

[43] X. Gloerfelt, C. Bailly, D. Juvé, Direct computation of the noise radiated by a subsonic cavity flow and application of integral methods, J. Sound Vib. 266 (1) (2003) 119-146.

[44] G.A. Bres, T. Colonius, Three-dimensional instabilities in compressible flow over open cavities, J. Fluid Mech. 599 (2008) $309-339$.

[45] K. Krishnamurty, Sound Radiation from Surface Cutouts in High Speed Flow (Ph.D. thesis), California Institute of Technology, 1956.

[46] L. Larchevêque, P. Sagaut, I. Mary, O. Labbé, P. Comte, Large-eddy simulation of a compressible flow past a deep cavity, Phys. Fluids 15 (1) (2003) 193-210.

[47] O. Guasch, R. Codina, Statistical behavior of the orthogonal subgrid scale stabilization terms in the finite element large eddy simulation of turbulent flows, Comput. Methods Appl. Mech. Engrg. 261 (2013) 154-166. 